
Aplicações na Síntese de Grupos Funcionais Nitrogenados

\author{
Araújo C. R. M.;* Gonsalves A. A.
}

Rev. Virtual Quim., 2015, 7 (4), 1469-1495. Data de publicação na Web: 29 de maio de 2015

http://www.uff.br/rvq

\title{
Oximes: Chemical Properties, Methods of Preparation and Applications in Synthesis of Nitrogen Functional Groups
}

\begin{abstract}
Oxime is an organic functional group having the following general formula $\mathrm{RR}^{\prime} \mathrm{C}=\mathrm{N}-\mathrm{OH}$ and although its unquestionable importance in the Coordination Chemistry and Organic Synthesis it is a subject underexplored by main chemistry textbooks in higher education adopted in Brazil. Thus, this article is a review about oximes organized in three parts. First are shown the properties of the oximes with emphasis in its isomerism and behavior in solution. After, are discussed some methods for obtaining oximes, such as oximation of carbonyl groups and double bonds, oxidation of amines, reduction of nitro compounds and the radical. Finally, in the third part of the article are treated some synthetic routes of nitrogen functional groups where oximes are precursors.
\end{abstract}

Keywords: Oxime; Chemical Properties of oximes; Synthesis of Oximes; Applications of Oximes in Organic Synthesis.

\section{Resumo}

Oxima é um grupo funcional orgânico que apresenta a fórmula geral $\mathrm{RR}^{\prime} \mathrm{C}=\mathrm{N}-\mathrm{OH}$ e apesar da sua relevância inquestionável na Química das Coordenações e na Síntese Orgânica este é um tema pouco explorado pelos principais livros de Química de ensino superior adotados no Brasil. Desta forma, o presente artigo trata de uma revisão sobre oximas, que está organizado em três partes. Inicialmente são mostradas as propriedades das oximas dando ênfase a sua isomeria e comportamento em solução. Em seguida, são abordadas algumas metodologias para a obtenção de oximas, como: oximação de carbonilas e duplas ligações, oxidação de aminas, redução de nitrocompostos e por via radicalar. Por fim, na terceira parte do artigo são tratadas algumas vias de síntese de grupos funcionais nitrogenados onde oximas são precursoras.

Palavras-chave: Oxima; Propriedades Químicas das Oximas; Síntese de Oximas; Aplicações de Oximas na Síntese Orgânica.

* Universidade Federal do Vale do São Francisco, Colegiado de Ciências Farmacêuticas, Campus Petrolina Centro, CEP 56304-917, Petrolina-PE, Brasil.

M cleonia.araujo@univasf.edu.br

DOI: $10.5935 / 1984-6835.20150080$ 


\section{Oximas: Propriedades Químicas, Métodos de Preparação e Aplicações na Síntese de Grupos Funcionais Nitrogenados}

\section{Cleônia Roberta M. Araújo, * Arlan A. Gonsalves}

Universidade Federal do Vale do São Francisco, Colegiado de Ciências Farmacêuticas, Campus Petrolina Centro, CEP 56304-917, Petrolina-PE, Brasil.

* cleonia.araujo@univasf.edu.br

Recebido em 19 de janeiro de 2015. Aceito para publicação em 29 de maio de 2015

\section{Introdução}

\section{Propriedades Químicas de Oximas}

\section{Métodos de Preparação de Oximas}

3.1. Síntese de oximas a partir de aldeídos e cetonas

3.2. Síntese de oximas a partir da adição de NOX a dupla ligação

3.3. Síntese de oximas a partir da oxidação de aminas primárias

3.4. Síntese de oximas a partir da redução de nitrocompostos

3.5. Síntese de oximas por via radicalar

\section{Oximas como Precursoras de Grupos Nitrogenados}

4.1. Obtenção de amidas via Rearranjo de Beckmann de oximas

4.2. Obtenção de anéis isozaxolínicos empregando oximas

4.3. Obtenção de nitrosos a partir de oximas

4.4. Obtenção de nitrocompostos através da oxidação de oximas

4.5. Obtenção de nitrilas através da desidratação de oximas

4.6. Obtenção de aminas através da redução de oximas

\section{Considerações Finais}

\section{Introdução}

As oximas compreendem um grupo funcional orgânico ainda pouco explorado pelos livros de química de ensino superior, apesar de sua grande importância para a Síntese Orgânica moderna. A química desta classe de compostos passou a ser investigada de forma mais significativa a partir de 1905, quando Lev Tschugaeff introduziu a dimetilglioxima como um reagente complexométrico seletivo e bastante sensível para a determinação gravimétrica de $\mathrm{Ni}(\mathrm{II}){ }^{1}$ Assim, as oximas conquistaram destaque como ligantes na química de coordenação, em razão da distribuição eletrônica peculiar do grupo, a qual proporciona formas distintas de ligação a cátions metálicos, ${ }^{2,3}$ Figura 1. 


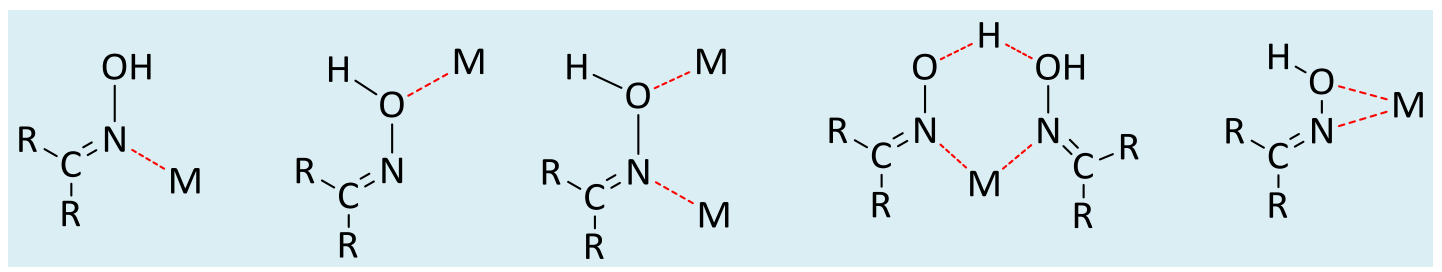

Figura 1. Formas distintas de complexação do grupo oxima com cátions metálicos. Adaptado de Kukushkin e Colaboradores (2003)

Durante o século XX e no atual, as oximas foram e continuam sendo estudadas por alguns pesquisadores, em decorrência principalmente da facilidade com que são sintetizadas, da grande variedade de matérias-primas disponíveis para sua preparação e de suas diferentes aplicações na indústria química e farmacêutica. Fazendo uma busca no Portal de Periódicos da CAPES é possível verificar que o número de artigos contendo o termo "oxime" no título cresceu mais de $100 \%$ comparando-se os anos de 2000 e 2014, ${ }^{4}$ Figura 2.

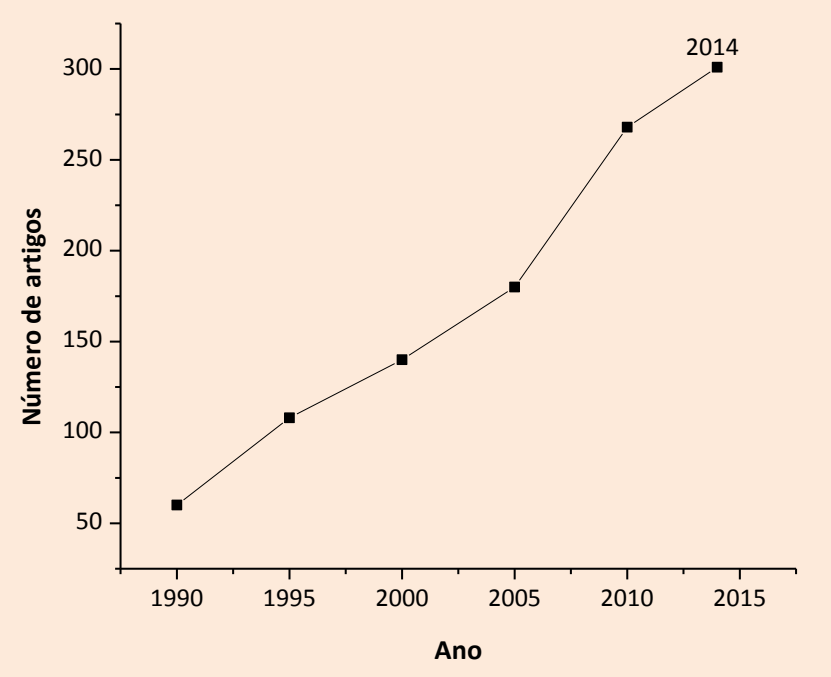

Figura 2. Número de artigos publicados contendo o termo "oxime" no título no portal de periódicos da CAPES entre os anos de 1990 e 2014

A utilização de oximas como precursores e intermediários na síntese de produtos naturais, industriais, fármacos e de agentes complexantes veem estimulando as pesquisas envolvendo esta classe de compostos nos últimos anos. ${ }^{5}$ Desta forma, visto a importância das oximas em algumas áreas da ciência, o presente artigo de revisão visa, inicialmente, descrever as propriedades químicas desta classe de compostos, um tema ainda pouco explorado pelos principais livros de Química de ensino superior adotados no Brasil, demonstrar uma série de métodos sintéticos para preparações deste grupo funcional e, por fim, expor algumas aplicações relevantes deste grupo funcional na síntese de compostos nitrogenados.

\section{Propriedades Químicas de Oximas}

O termo oxima foi primeiramente definido 
no século XIX, sendo provavelmente resultante da contração das palavras oxigênio e imina. ${ }^{6}$ As iminas ou bases de Schiff são moléculas orgânicas que apresentam fórmula molecular geral RR'C=NR" e são obtidas a partir de reações de condensação entre aldeídos ou cetonas com compostos que apresentam o grupo $-\mathrm{NH}_{2}$, tais como: aminas primárias $\left(\mathrm{RNH}_{2}\right)$, hidroxilaminas $\left(\mathrm{NH}_{2} \mathrm{OH}\right)$, hidrazinas $\left(\mathrm{NH}_{2} \mathrm{NH}_{2}\right)$, e semicarbazidas $\left(\mathrm{NH}_{2} \mathrm{NHCONH}_{2}\right)^{7}$. Desta forma, as oximas são consideradas compostos análogos das iminas, ${ }^{8}$ onde $\mathrm{R}^{\prime \prime}=-\mathrm{OH}$, o que resulta na seguinte fórmula geral: $\mathrm{RR}^{\prime} \mathrm{C}=\mathrm{N}-\mathrm{OH} .{ }^{9}$ Trata-se de compostos químicos estáveis e de fácil preparação, ${ }^{10}$ sendo classificadas em aldoximas e cetoximas dependendo da natureza dos radicais $R$ e $R^{\prime}$ em sua estrutura. As cetoximas apresentam duas cadeias laterais alquila ( $R$ e $R^{\prime}$ ), já nas aldoximas, um destes dois radicais é obrigatoriamente um átomo de hidrogênio, ${ }^{11}$ Figura 3.

Em razão da geometria da dupla ligação $\mathrm{C}=\mathrm{N}$ existente nas oximas $\left(\mathrm{RR^{ \prime }} \mathrm{C}=\mathrm{N}-\mathrm{OH}\right)$ estas apresentam estereoisomeria do tipo $E$ e $Z .^{8}$ Aplicando a convenção de Cahn-IngoldPrelog, quando o grupo alquila de maior prioridade se encontrar do mesmo lado que a hidroxila, o isômero será do tipo $Z$ (syn) e, quando este se encontrar em posição oposta, o isômero será do tipo $E$ (anti). ${ }^{12}$ Além de diferentes propriedades químicas, os isômeros de oximas também podem apresentar propriedades biológicas diferentes, como ocorre com a fluvoxamina (Figura 4), um éter de oxima inibidor da recaptação de serotonina utilizado para o tratamento da depressão e da doença obsessivo-compulsiva, cujo isômero $E$ é o único que exibe atividade farmacológica, ${ }^{13}$ Figura 3.

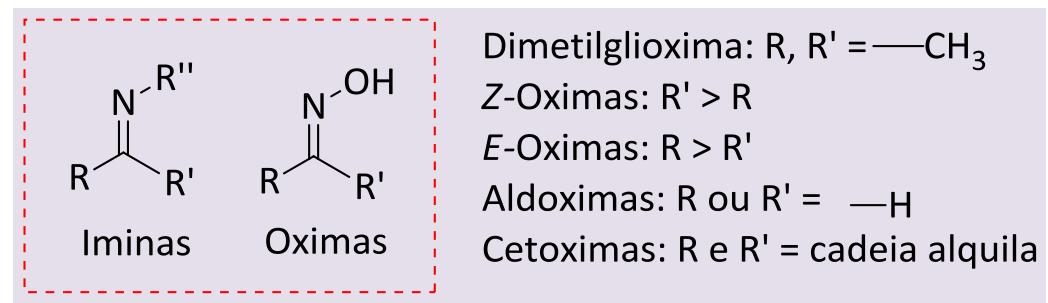

Figura 3. Dimetilglioxima e fórmula molecular geral de iminas, oximas $(Z$ e $E)$, aldoximas e cetoximas

Compostos derivados de oximas também estão amplamente relatados na literatura, sendo alguns deles utilizados como fármacos. Os derivados mais comuns são: éteres de oximas (RR' $\left.\mathrm{C}=\mathrm{N}-\mathrm{O}-\mathrm{R}^{\prime \prime}\right)$ e amidoximas $\left(R\left(N R^{\prime} R^{\prime \prime}\right) C=N-O-R^{\prime \prime \prime}\right)$. A Figura 4 demonstra alguns fármacos que contém o grupo oxima ou derivados em sua estrutura química.

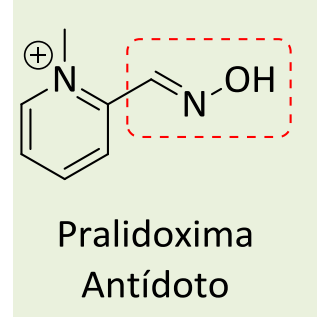

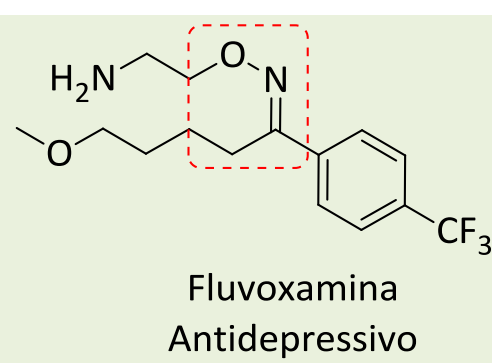

Figura 4. Estruturas químicas de fármacos contendo o grupo oxima (pralidoxima) e éter de oxima (fluvoxamina) 
A acetaldoxima, uma das oximas mais simples que exibe isomeria syn/anti, encontra-se na forma de uma mistura de estereoisômeros $E$ e $Z$. Nas fases de vapor e na líquida, a população do isômero $E$ compreende, respectivamente, cerca de 40 e $37 \%$ da mistura. ${ }^{13-15}$ Contudo, no estado sólido, a população $E / Z$ depende da forma de preparação do composto, ${ }^{16}$ da estrutura da molécula e do solvente utilizado para a recristalização. ${ }^{17} \mathrm{Em}$ 2006, Nsikabaka e Colaboradores $^{13}$ realizaram cálculos teóricos para estudar a interconversão em fase aquosa entre os isômeros $E$ e $Z$ da acetaldoxima. Segundo os autores deste trabalho, a isomerização é promovida em meio ácido e através da rotação da ligação $\mathrm{C}=\mathrm{N}$ da oxima, com geração prévia de uma ligação reversível $\mathrm{C}_{\text {oxima }}-\mathrm{O}_{\text {água }}$ para estabilizar o sistema. Nesse caso, a água atua como um catalisador nucleofílico e o processo não envolve a quebra de ligações $\mathrm{O}-\mathrm{H}$ do grupo oxima. Ainda segundo os autores, este mecanismo de interconversão isomérica pode ser válido para outras oximas solúveis em água. A Esquema 1 demonstra o mecanismo de isomerização $E / Z$ da acetaldoxima em fase aquosa.

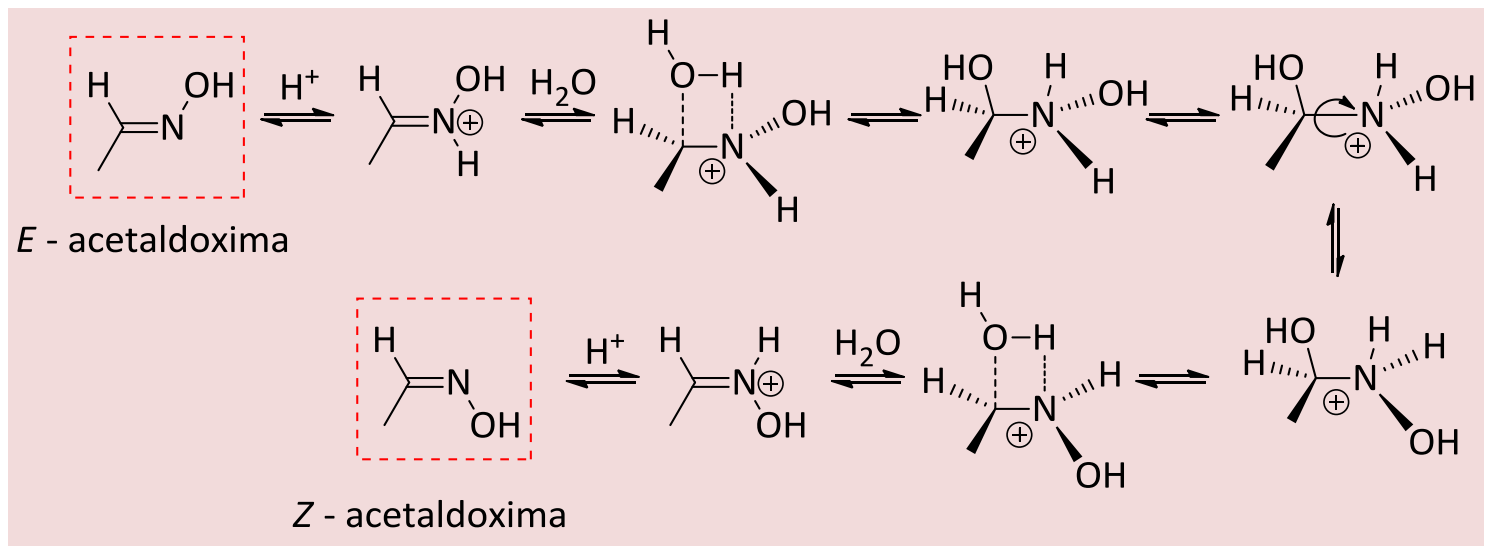

Esquema 1. Mecanismo de interconversão entre os isômeros $E$ e $Z$ da acetaldoxima em meio aquoso ácido. Adaptado de Nsikabaka e Colaboradores (2006) ${ }^{13}$

Uma propriedade decorrente da isomeria $E / Z$, sugerida por Golec e Colaboradores, ${ }^{16}$ é que a maior habilidade do isômero $Z$ da acetaldoxima em produzir fortes ligações de hidrogênio com moléculas de nitrogênio gasoso $\left(\mathrm{N}_{2}\right)$, em comparação com o isômero $E$, sugere que o grupo $-\mathrm{OH}$ desta oxima tende a apresentar um caráter mais ácido na estereoisomeria $Z$. Os isômeros geométricos $E / Z$ de iminas são na maioria instáveis e se interconvertem rapidamente em temperatura ambiente. Por outro lado, os estereoisômeros $E / Z$ de oximas são estáveis o suficiente para serem isolados da mistura. ${ }^{18}$

Compostos contendo o grupo oxima são anfóteros, uma vez que podem revelar um caráter levemente ácido, em função do hidrogênio ionizável no grupo hidroxila, e uma discreta basicidade, devido à presença do par de elétrons livres no átomo de nitrogênio. ${ }^{19}$ O comportamento de ácido fraco de oximas confere valores de $\mathrm{p} K_{a}$ que variam de forma significativa de acordo com a estrutura química do composto (Tabela 1). Estudos também sugerem que as cetoximas se comportam como ácidos mais fracos que as aldoximas correspondentes. ${ }^{20}$ As oximas são estáveis em condições alcalinas, e este meio favorece a formação da base conjugada deste grupo, o ânion oximato $\left(\mathrm{R} \mathrm{R}^{\prime} \mathrm{C}=\mathrm{N}-\mathrm{O}^{\Theta}\right){ }^{20}$ 
Tabela 1. Valores de constante de acidez (Ka) em p-valor de algumas oximas

PKa

Em solução, as oximas apresentam-se como uma mistura de dois compostos estruturalmente distintos que se encontram em equilíbrio tautomérico, o qual é observado entre as funções nitroso e oxima. ${ }^{23,24} \mathrm{O}$ tautomerismo oxima $\rightleftharpoons$ nitroso pode ser comparado a outros equilíbrios tautoméricos, tais como: amida $\rightleftharpoons$ amidol, imina $\rightleftharpoons$ enamina, ceto $\rightleftharpoons$ enólico e nitro $\rightleftharpoons$ aci-nitro. $^{25} 0$ nitroso participante desse tautomerismo será estável caso não exista um hidrogênio ligado ao carbono componente do grupo oxima pois, quando isso ocorrer, o equilíbrio químico estará mais deslocado no sentido de formar a oxima, que geralmente passa a ser o produto mais estável. ${ }^{23,24} \mathrm{Em}$ alguns casos entretanto, mesmo com a presença do hidrogênio tautomerizável na molécula, a interconversão nitroso $=$ oxima não ocorre espontaneamente, mas ainda assim, pode ser promovida através de catálise ácida ou básica em solventes próticos. Uma explicação para este fato é que a facilidade de tautomerização também depende, em certa extensão, da estrutura química do composto, ${ }^{26}$ Figura 6.

Em razão da tautomerização, após o aquecimento brando de soluções orgânicas de oximas, principalmente em solventes de baixa constante dielétrica como clorofórmio $\left(\mathrm{CHCl}_{3}\right)$ e diclorometano $\left(\mathrm{CH}_{2} \mathrm{Cl}_{2}\right)$, estas tendem a apresentar uma coloração azulada ou esverdeada, sugerindo o favorecimento da formação do monômero de nitroso no meio. À temperatura ambiente, em geral, compostos nitrosos estáveis se apresentam como sólidos brancos, com estrutura química de dímeros de dióxido de diazeno, na maioria dos casos em isomeria trans, e cujas soluções são incolores, ${ }^{25,27,28}$ Esquema 2. 


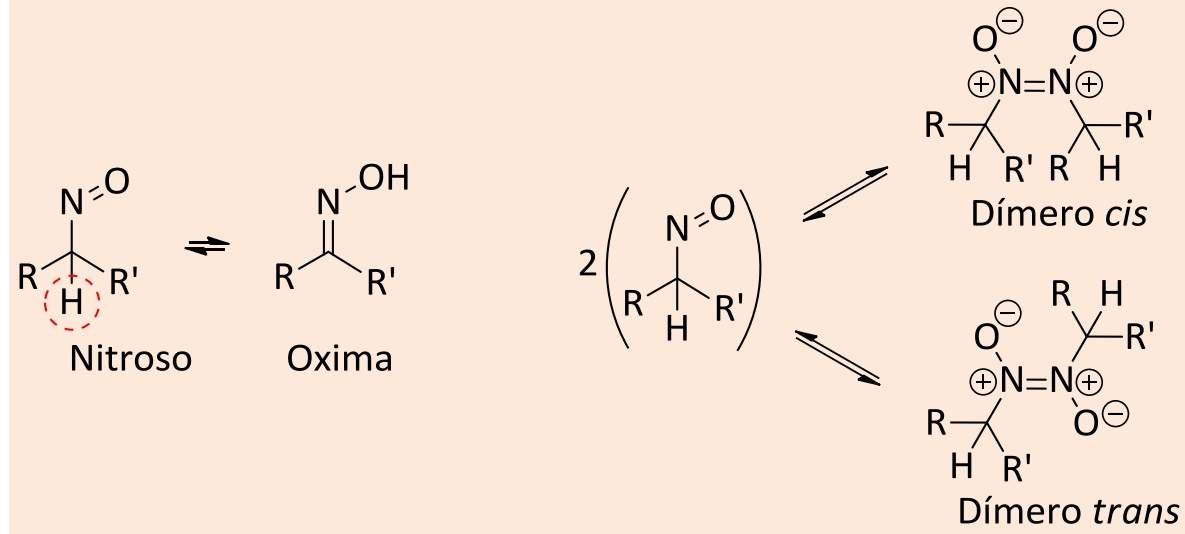

Esquema 2. Equilíbrio tautomérico nitroso $\rightleftharpoons$ oxima e possíveis formas diméricas de compostos nitrosos estáveis

Em 1996, Glaser e Colaboradores ${ }^{28}$ explicaram através de cálculos computacionais de estabilidade termodinâmica o motivo das oximas não se apresentarem na forma dimérica, a exemplo do que normalmente ocorre com seus respectivos nitrosos tautoméricos estáveis. Após a realização dos experimentos foi observado que a dimerização de oximas leva à formação de um composto com elevada densidade eletrônica sobre o oxigênio, o que resulta em uma polarização reversa desfavorável sobre o átomo de carbono. Ao observar as estruturas de Kekulé dos dímeros de nitroso (N1, N2 e N3) e oxima (O1, O2 e O3) é possível notar que $\mathrm{O} 2$ e $\mathrm{O} 3$ desfavorecem a formação do dímero de oxima em virtude da presença de carbonos deficientes em elétrons nessas estruturas. Em contrapartida, as estruturas N2 e N3 estabilizam o dímero de nitroso, ${ }^{28}$ Esquema 3.

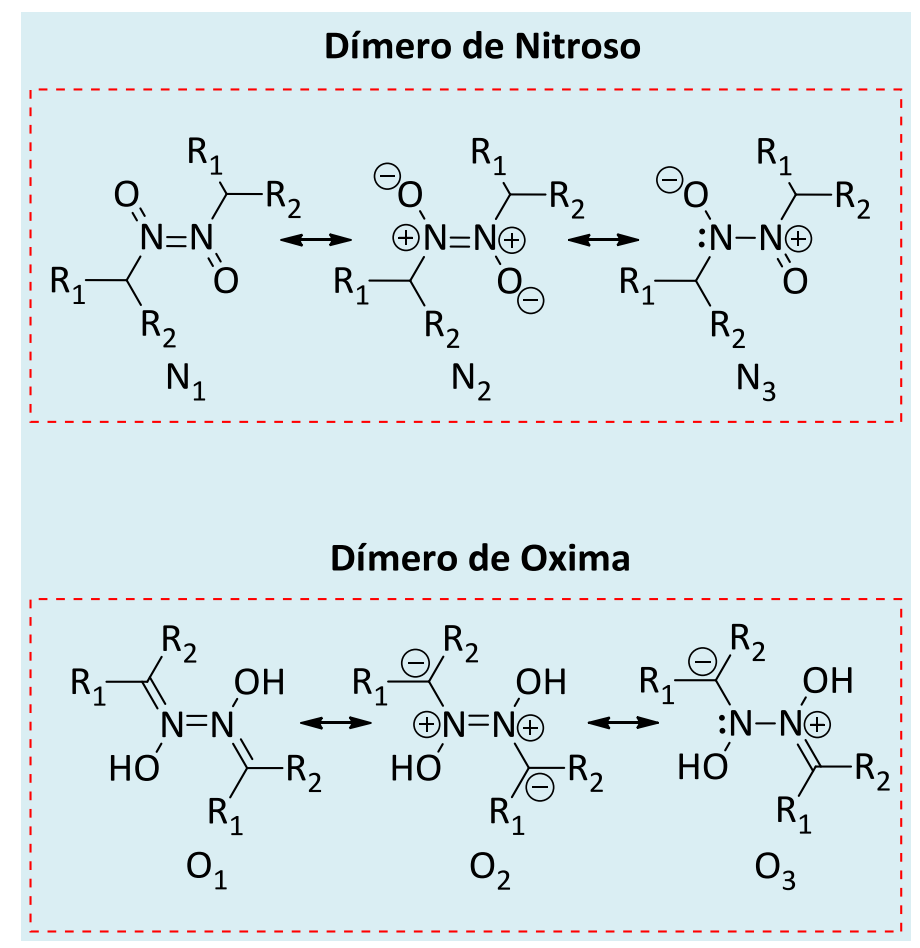

Esquema 3. Estruturas de Kekulé dos dímeros de nitroso (N1, N2 e N3) e de oxima (O1, O2 e O3). Adaptado de Glaser e Colaboradores $(1996)^{28}$ 
$\mathrm{Na}$ identificação espectroscópica de oximas, seu espectro no ultravioleta próximo mostra uma banda com máximo de absorção entre 220 e $230 \mathrm{~nm}$, referente à transição $\pi \rightarrow \pi^{*}$ (banda K) do cromóforo de oxima $(\mathrm{C}=\mathrm{N}) .{ }^{29}$ Espectros de infravermelho revelam vibrações de deformação axial $v_{C=N}$ em 1597 $\mathrm{cm}^{-1}, \mathrm{U}_{\mathrm{N}-\mathrm{O}}$ em $1005 \mathrm{~cm}^{-1}$ e $\mathrm{U}_{\mathrm{O}-\mathrm{H}}$ em $3310 \mathrm{~cm}^{-}$ ${ }^{1.30}$ Espetros de ressonância magnética nuclear de hidrogênio $\left({ }^{1} \mathrm{H} R M N\right)$ revelam um singleto com deslocamento químico entre $\delta$ 10,9 e $\delta$ 12,4 ppm, correspondente à absorção do hidrogênio-1 do grupo oxima $\left(R^{\prime} \mathrm{C}=\mathrm{N}-\mathrm{O}-\mathrm{H}\right) .{ }^{31}$ Já os espectros de ${ }^{13} \mathrm{C} \mathrm{RMN}$ de oximas demonstram absorção entre $\delta 145$ e $\delta 165 \mathrm{ppm}$, correspondente ao carbono-13 alílico, sendo comum a distinção entre os sinais dos isômeros syn e anti. No espectro de ${ }^{15} \mathrm{~N}$ RMN estes compostos exibem absorção entre $\delta 330$ e $\delta 380$ ppm, a qual corresponde à absorção do isótopo de nitrogênio-15 do grupo oxima. ${ }^{32}$

\section{Métodos de Preparação de} Oximas

A relevância do grupo oxima na Síntese Orgânica está relacionada com a versatilidade desta classe de compostos, visto que as oximas podem ser obtidas a partir de uma série de grupos funcionais, tais como: aldeídos, $^{33}$ cetonas, $^{33}$ olefinas, ${ }^{34}$ aminas, $^{35}$ nitrocompostos, $^{36}$ nitritos $^{37}$ e compostos que apresentam hidrogênios $\alpha$ carbonila. ${ }^{24}$ Grupos nitrogenados, como: amidas, $^{39}$ anéis isoxazolínicos, ${ }^{40}$ nitrosos, ${ }^{41}$ nitrocompostos, ${ }^{42}$ nitrilas ${ }^{43}$ e aminas ${ }^{44}$ podem ser preparados a partir de oximas, Esquema 4.

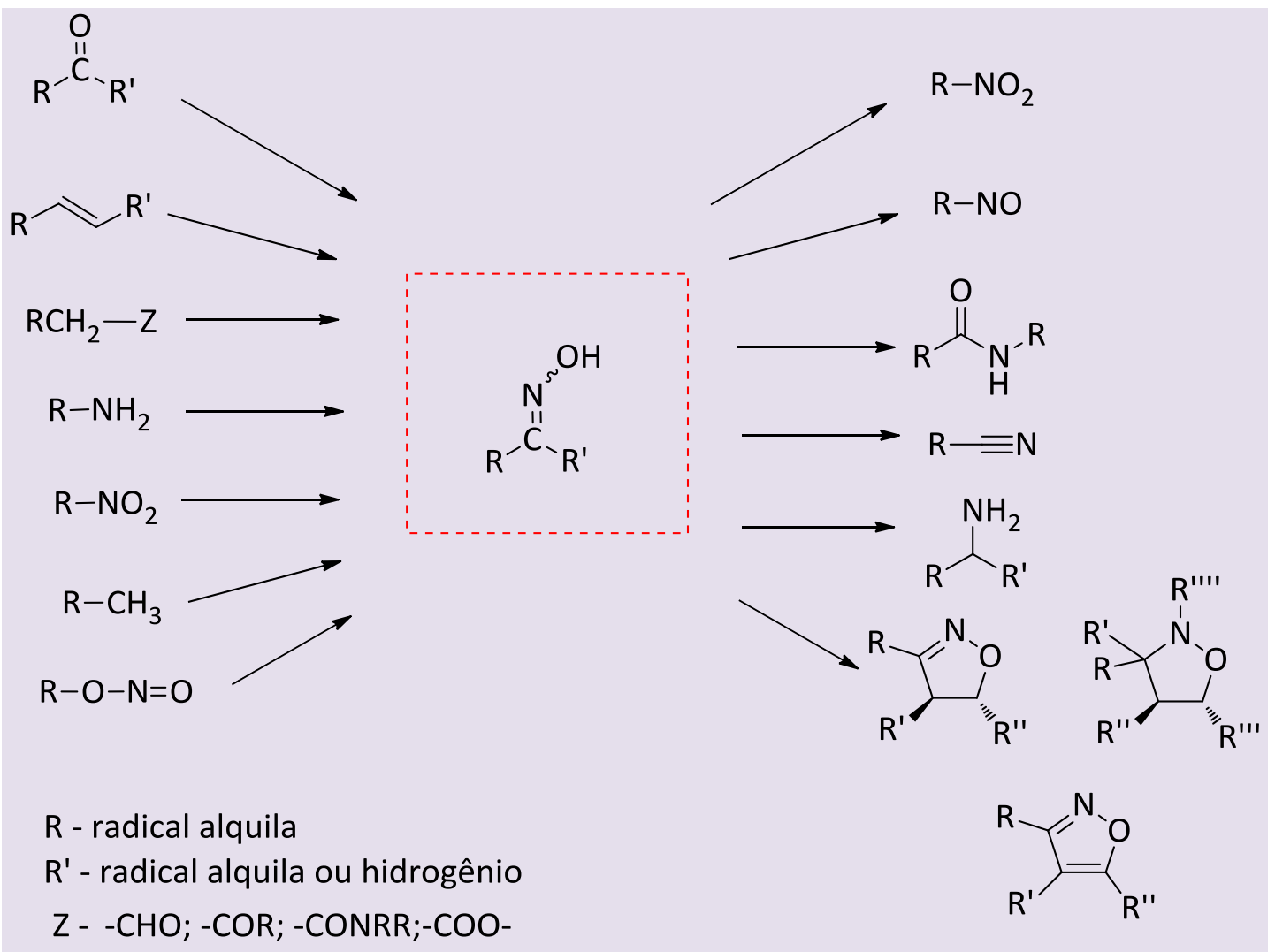

Esquema 4. Grupos funcionais que formam oximas e grupos funcionais que podem ser preparados a partir de oximas 


\subsection{Síntese de oximas a partir de aldeídos e cetonas}

O método de síntese mais importante e utilizado para a preparação de oximas é a partir da adição de hidroxilamina $\left(\mathrm{NH}_{2} \mathrm{OH}\right)$ a aldeídos e cetonas. ${ }^{33,45,46}$ As oximas obtidas a partir destas matérias-primas são denominadas de aldoximas e cetoximas, respectivamente. A reação ocorre em duas etapas, iniciando pelo ataque da hidroxilamina à carbonila. A carbinolamina formada desidrata gerando a oxima correspondente, ${ }^{47}$ Esquema 5.

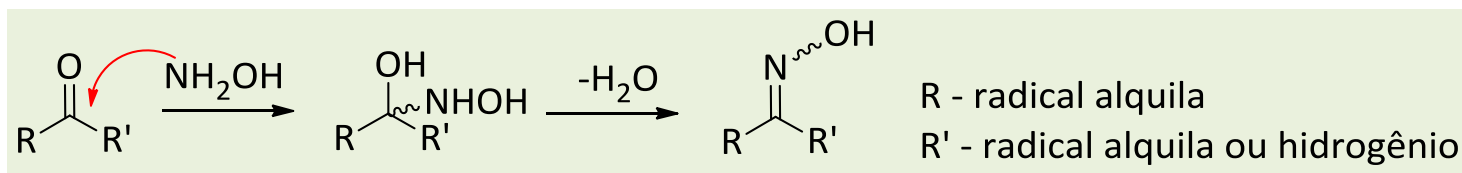

Esquema 5. Reação de cetonas ou aldeídos com hidroxilamina para a formação de oximas

Nesta metodologia a taxa de formação da oxima depende do $\mathrm{pH}$ do meio, sendo o $\mathrm{pH} 4$ o ideal. O aumento ou diminuição deste provoca um decréscimo na geração da oxima. $\mathrm{Em}$ pH abaixo de 4 a segunda etapa da reação (desidratação) ocorre rapidamente, pois esta é catalisada por ácidos. Contudo, a primeira etapa (ataque nucleofílico) é reduzida, visto que em condições ácidas ocorre o equilíbrio entre a $\mathrm{NH}_{2} \mathrm{OH}$ e seu ácido conjugado $\left[\mathrm{NH}_{3} \mathrm{OH}\right]^{+}$, que não ataca o substrato. $\mathrm{O} \mathrm{pH} 4$ favorece o equilíbrio em direção à $\mathrm{NH}_{2} \mathrm{OH}$ e este alcança sua concentração máxima, levando ao aumento na velocidade da primeira etapa e redução da segunda, desta forma, a etapa limitante da reação é a segunda e a elevação do $\mathrm{pH}(\mathrm{pH}>$ 4) provoca uma redução na taxa de reação global. ${ }^{48}$

Muitas melhorias desta metodologia foram realizadas. Ballini e Colaboradores utilizaram uma resina de troca iônica (Amberlyst A-21) para catalisar a reação de compostos carbonílicos e cloridrato de hidroxilamina $\left(\mathrm{NH}_{2} \mathrm{OH} . \mathrm{HCl}\right)$ e obtiveram bons rendimentos. ${ }^{49}$ Alumina básica, $\mathrm{TiO}_{2}, \mathrm{CaO}$ e compostos de bismuto também vêm sendo empregados na preparação de oximas sob radiação de micro-ondas na ausência ou não de solvente. ${ }^{50}$

\subsection{Síntese de oximas a partir da adição de NOX à dupla ligação}

A reação entre olefinas e cloreto de nitrosila $(\mathrm{NOCl})$ para gerar $\alpha$-cloro oxima é conhecida desde 1875, sendo essa metodologia usada para a identificação de estruturas moleculares de terpenos. ${ }^{51,52}$ Neste caso, outros grupos funcionais como ácidos carboxílicos, ésteres, nitrilas e éteres podem estar presentes na molécula e não interferir na síntese da $\alpha$-cloro oxima. Vários trabalhos mostram detalhes da reação utilizando alcenos, cicloalcenos, cetenos, acetais, viniléteres, cetona $\alpha$ 国 $\beta$-insaturadas contendo um substituinte no grupo vinílico, cloroalcenos, derivados ciclopropenos e enaminas. ${ }^{34}$

O NOX é o agente nitrosante dessa metodologia e a reação é iniciada pela adição do cátion $\mathrm{NO}^{+}$à dupla ligação. $\mathrm{O}$ intermediário gerado é atacado pelo ânion do halogênio $\left(X^{-}\right)$e, por fim, o $\beta$-halo nitroso formado sofre tautomerização para originar a oxima correspondente. A reação de adição do NOX à dupla ligação pode ter isomeria cis ou trans, visto que o intermediário é um carbocátion com geometria triangular plana. Dessa forma, o ataque nucleofílico pode 
acontecer em ambos os lados. O produto da reação, em geral, obedece a regioquímica de Markovnikov, visto que o carbocátion formado preferencialmente, o mais substituído, será o intermediário mais estável, ${ }^{23,53,54}$ Esquema 6.

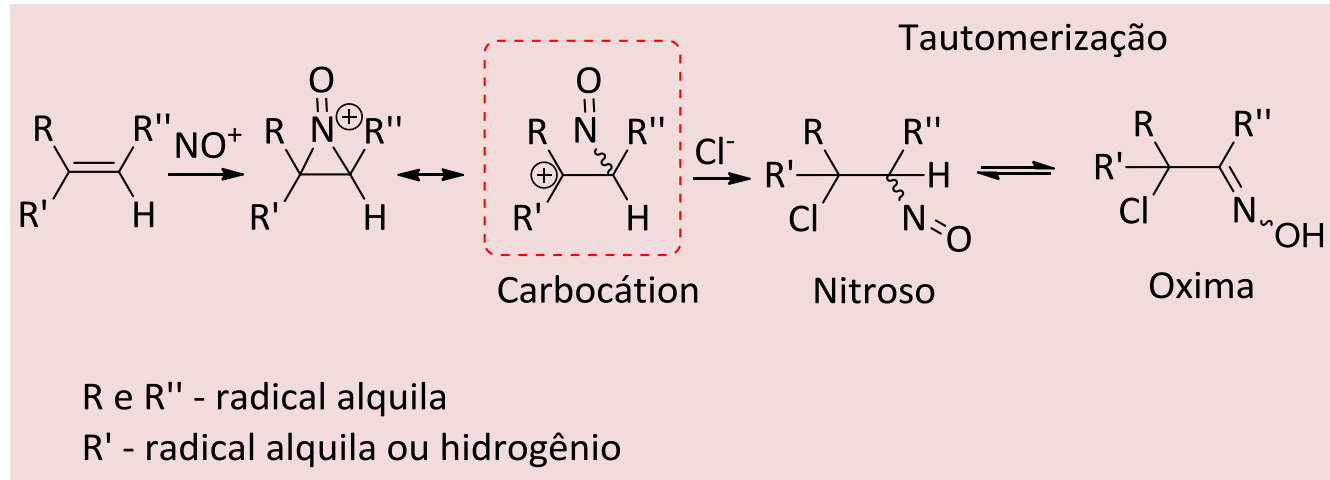

Esquema 6. Reação de alcenos e NOX para gerar oximas

O $\beta$-halo nitroso formado pode sofrer dimerização, com formação da ligação N-N, e estabelecer um equilíbrio entre a forma monomérica e dimérica. Apenas o monômero sofre tautomerização irreversível para a oxima correspondente mais estável que o nitroso de origem. Esse é um processo não espontâneo catalisado por ácidos ou bases. $^{26}$

Carbonos $\alpha$-carbonila, também chamados de carbonos enolizáveis, vizinhos de grupos como: OOR; CHO; COR; CONRR e COO', são susceptíveis à nitrosação, de forma análoga aos alcenos. Estes compostos apresentam hidrogênios ácidos e, em solução, encontram-se em equilíbrio tautomérico. ${ }^{38} \mathrm{~A}$ forma enólica reage com NOX, sendo a reação provavelmente iniciada pela adição do $\mathrm{NO}^{+}$à dupla ligação do enol o que gera o intermediário nitroso que, em seguida, perde o próton restaurando a carbonila. Por fim, a cetoxima é formada após a tautomerização do $C$-nitroso, ${ }^{24,34}$ Esquema 7.

Nesta metodologia de obtenção de oximas, a espécie NOX é fundamental, e esta pode ser gerada a partir de nitritos de alquila (RONO) ou ácido nitroso (HONO), em presença de ácido, segundo a seguinte equação: RONO + XH $\rightarrow \mathrm{NOX}+\mathrm{ROH} .{ }^{55,56,57}$

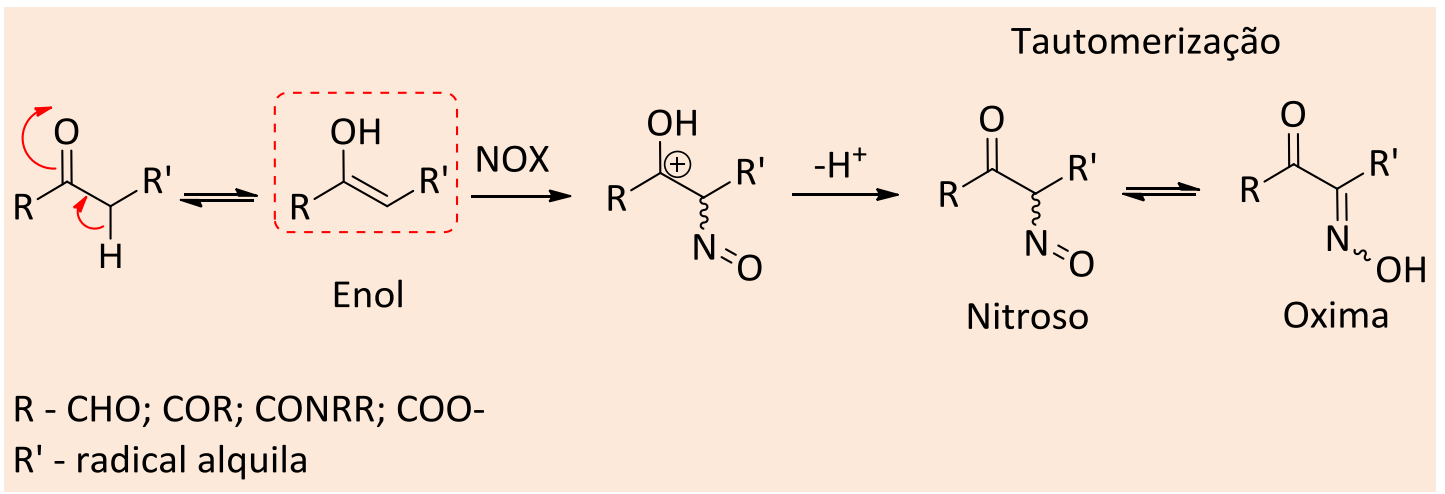

Esquema 7. Reação de preparação de oximas em carbonos $\alpha$-carbonila (carbonos enolizáveis) 
3.3. Síntese de oximas a partir da oxidação de aminas primárias

Aminas primárias alifáticas podem ser oxidadas para gerar oximas, desta forma, dentre os agentes de oxidação utilizados estão: o ácido de Caro $\left(\mathrm{H}_{2} \mathrm{SO}_{5}\right){ }^{35}$ o ácido peracético $\left(\mathrm{H}_{2} \mathrm{O}_{2}\right.$ em ácido acético $){ }^{58}$ o ácido 3-cloroperbenzoico, o perborato de sódio ${ }^{59} \mathrm{e}$ - $\mathrm{Na}_{2} \mathrm{WO}_{4}-\mathrm{H}_{2} \mathrm{O}_{2} \cdot{ }^{60}$ Ao utilizar o ácido de Caro a reação é iniciada pela formação da hidroxilamina que sofre oxidação e origina o nitroso após desidratação. Por fim, este tautomeriza para a oxima correspondente segundo a Esquema $8 .{ }^{61}$

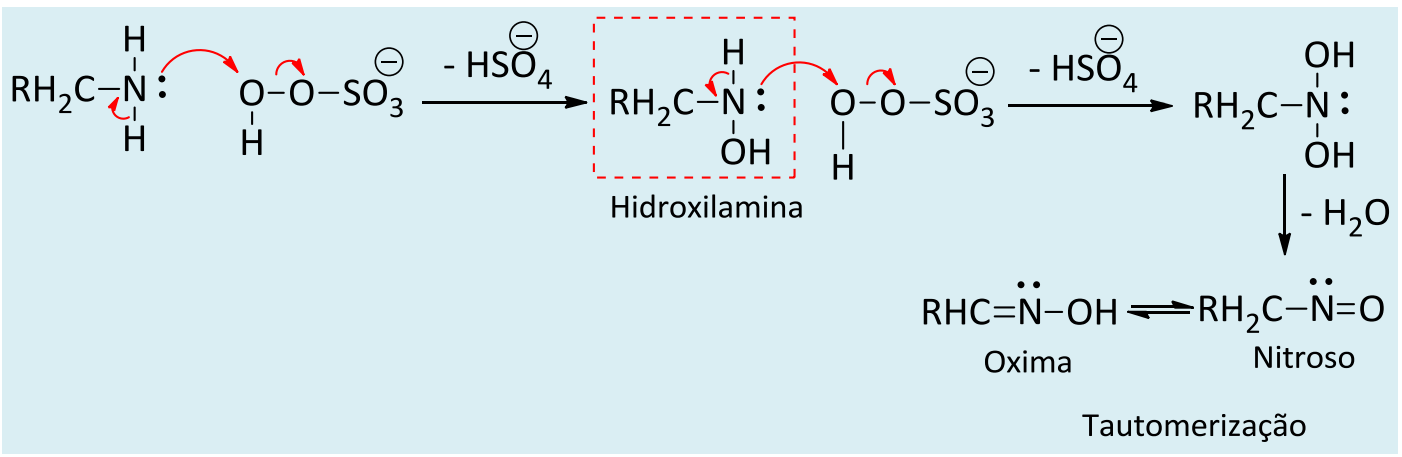

Esquema 8. Reação de oxidação de aminas primárias a oximas usando o ácido de Caro

Bamberger e Seligman foram os primeiros a utilizar ácido de Caro para produzir nitrosoalcanos a partir das seguintes aminas primárias $\left(\mathrm{CH}_{3}\right)_{2} \mathrm{CH}-\mathrm{CHRNH}_{2}(\mathrm{R}=\mathrm{H}$, Me ou COMe). Os nitrosocompostos preparados após tautomerização originaram as oximas correspondentes, $\left(\mathrm{CH}_{3}\right)_{2} \mathrm{CH}-\mathrm{CR}=\mathrm{NOH}$, onde $\mathrm{R}$ $=\mathrm{H}, \mathrm{Me}$ ou $\mathrm{COMe}^{34}$ Emmons preparou nitrosos através da oxidação de aminas primárias com ácido peracético, o qual foi obtido a partir de $\mathrm{H}_{2} \mathrm{O}_{2}$ e anidrido acético em diclorometano. ${ }^{62}$ Gilbert e Borden oxidaram a 2-butilamina, a 1-hexilamina, a 1propilamina, a 2-feniletilamina e a ciclohexilamina com ácido 3cloroperbenzoico e obtiveram os dímeros de nitroso correspondentes que, após tautomerização, geraram as respectivas oximas com bons rendimentos. ${ }^{63}$

Tollari e Porta empregaram o complexo $\mathrm{MoO}\left(\mathrm{O}_{2}\right)_{2}\left(\mathrm{H}_{2} \mathrm{O}\right)(\mathrm{HMPA})$ para oxidar aminas aromáticas, $\mathrm{RCH}_{2} \mathrm{NH}_{2}\left(\mathrm{R}=\mathrm{Ph}, 2,4-\mathrm{Cl}_{2} \mathrm{C}_{6} \mathrm{H}_{3}\right.$, 2$\mathrm{ClC}_{6} \mathrm{H}_{4}$ ou 3,4-(OMe $\left.{ }_{2} \mathrm{C}_{6} \mathrm{H}_{3}\right)$, e gerar as respectivas oximas. ${ }^{64} \mathrm{Em} \mathrm{2011,} \mathrm{Kidwai} \mathrm{e}$ Bhardwaj obtiveram oximas a partir da oxidação de aminas com $\mathrm{H}_{2} \mathrm{O}_{2}$ e os seguintes catalisadores: $\mathrm{SiO}_{2} ; \quad \mathrm{ZrO}_{2} ; \quad \mathrm{Al}_{2} \mathrm{O}_{3} ; \mathrm{Ti}\left(\mathrm{O}^{i} \mathrm{Pr}\right)_{4}$;
$\mathrm{Nb}_{2} \mathrm{O}_{5} ; \mathrm{TiO}_{2} \cdot{ }^{65}$ Oxigênio molecular em associação com 1,1-difenil-2-picrilhidrazil (DPPH) e óxido de tungstênio/alumina $\left(\mathrm{WO}_{3} / \mathrm{Al}_{2} \mathrm{O}_{3}\right)$ foram empregados por Suzuki para sintetizar oximas em 2013. ${ }^{66}$

\subsection{Síntese de oximas a partir da redução de nitrocompostos}

Nitrocompostos que contêm hidrogênio $\alpha$ podem ser reduzidos a oximas empregando zinco metálico em ácido acético ou outros reagentes, como: $\mathrm{CS}_{2}-\mathrm{Et}_{3} \mathrm{~N}, \mathrm{CrCl}_{2}$ e $\mathrm{NaNO}_{2}$. ${ }^{36,61}$ Hwu e Colaboradores preparam cetoximas alifáticas e aromáticas com bons rendimentos utilizando nitroalcanos secundários e hexametilsilano em meio básico. Um mecanismo plausível para esta reação é mostrado na Figura 13. Neste processo, o nitronato é gerado inicialmente empregandose uma base forte. $O$ íon nitronato ataca 0 hexametilsilano para formar o silil nitronato e o ânion $\mathrm{Me}_{3} \mathrm{Si}^{-}$. Em seguida, o centro nucleofílico do silil nitronato é atacado pelo $\mathrm{Me}_{3} \mathrm{Si}^{-}$e a silil oxima é gerada após a 1,2-syn- 
eliminação. A silil oxima sofre novo ataque do a cetoxima, ${ }^{67}$ Esquema 9.

$\mathrm{Me}_{3} \mathrm{Si}^{-}$e o oximato é protonado para produzir

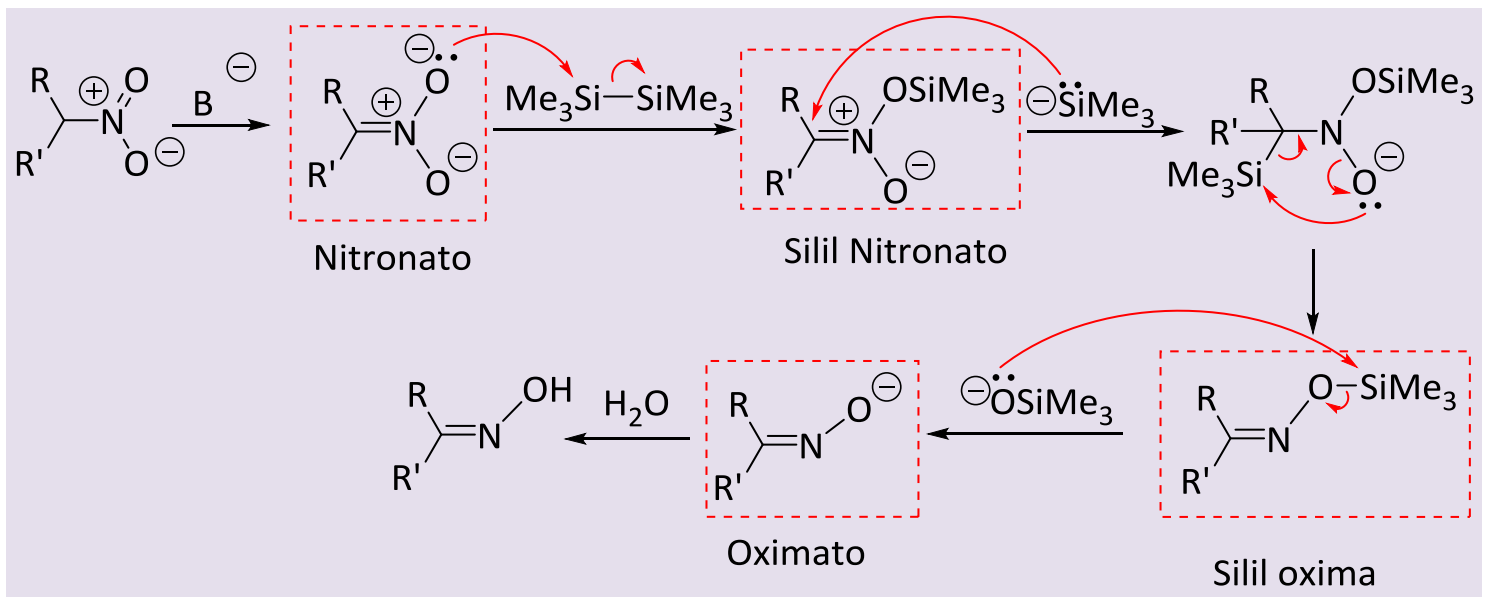

Esquema 9. Síntese de oximas a partir de nitroalcanos e hexametilsilano em meio básico. Adaptado de Hwu e Colaboradores (1999) ${ }^{67}$

Reagentes de Grinard podem ser empregados na obtenção de cetoximas. Nessa reação a base conjugada de nitrocompostos, formada pelo tratamento do nitroalcanos com $n$-BuLi, reagem com Grinard em presença de $\mathrm{ClCH}=\mathrm{NMe}_{2}{ }^{+} \mathrm{Cl}^{-}$para produzir cetoximas. ${ }^{12}$

\subsection{Síntese de oximas por via radicalar}

Nitrosaminas podem gerar amino oximas através de fotoadição com olefinas em meio ácido, solventes próticos e sob radiação ultravioleta. Nesta reação, ocorre inicialmente a quebra homolítica da ligação $\mathrm{N}-\mathrm{N}$ e a formação do cátion radical $\left(\mathrm{RR}^{\prime} \mathrm{N}-\mathrm{H}\right)^{\circ+}$ e do radical •NO. Em seguida, as espécies sofrem adição à dupla ligação para produzir um intermediário nitroso. O amino nitroso gerado tautomeriza para a $\alpha$-amino oxima correspondente, ${ }^{34,68}$ Esquema 10.

Nitritos orgânicos podem sofrer fotólise e gerar hidroxi-oximas. Nesta reação o nitrito é fotoexcitado à 1,2-dirradical, o qual sofre fragmentação gerando ${ }^{\circ} \mathrm{NO}$ e radical alcoóxido. Em seguida, ocorre uma transferência intramolecular de um átomo de hidrogênio do tipo 1,5. O radical alquila formado captura o radical NO anteriormente produzido na fotólise gerando o nitroso que tautomeriza para a oxima correspondente. Esta é a chamada reação de Barton e é empregada para a funcionalização de hidrocarbonetos, especialmente os esteroides, ${ }^{37,69,70}$ Esquema 11.

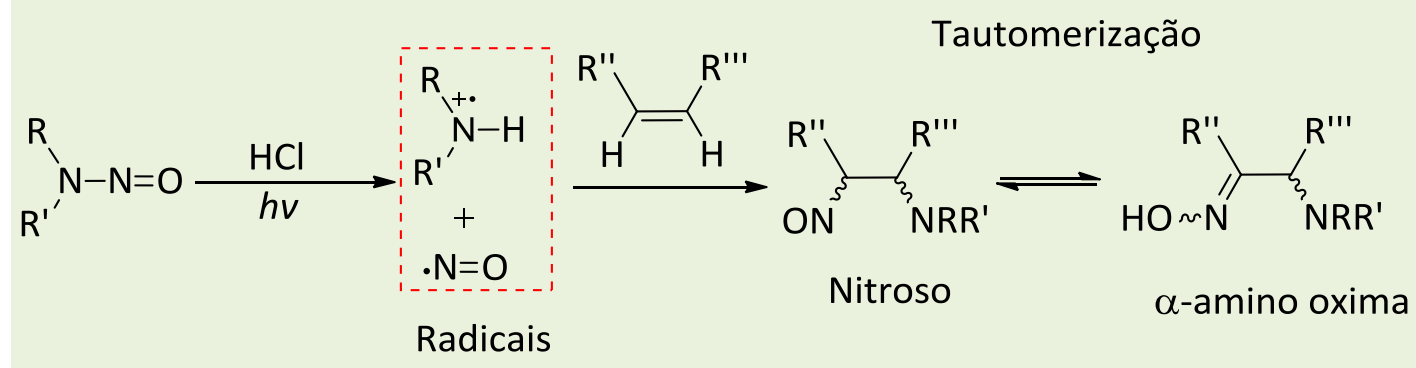

Esquema 10. Síntese de $\alpha$-amino oximas a partir de nitrosaminas e alcenos 


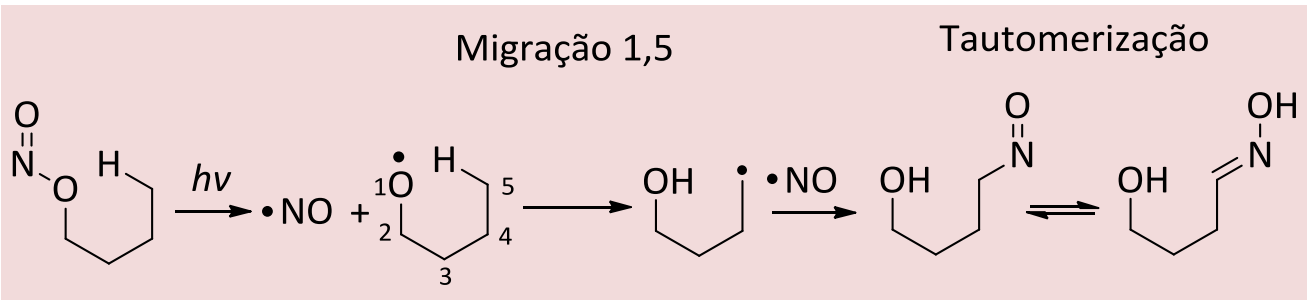

Esquema 11. Síntese de oximas a partir da fotólise de nitritos orgânicos. Adaptado de Akhtar e Pechet $(1965)^{69}$

\section{Oximas como Precursoras de Grupos Nitrogenados}

Na Síntese Orgânica as oximas merecem menção por sua versatilidade, as quais podem ser obtidas por uma série de reações químicas e atuar na preparação de vários grupos nitrogenados. Neste último aspecto, destaca-se o Rearranjo de Beckmann, reação empregada na síntese industrial de lactamas, ${ }^{71}$ e as reações de cicloadição 1,3dipolar, onde os anéis isoxazolínicos produzidos são promissores para a Química Medicinal $^{72,73}$ e para o desenvolvimento de novos materiais tecnológicos. ${ }^{74,75}$

\subsection{Obtenção de amidas via Rearranjo de} Beckmann de oximas

As oximas, em presença de ácidos próticos, ácidos de Lewis, anidridos e haletos de acila, sofrem rearranjo para gerar amidas, sendo este processo chamado de Rearranjo de Beckmann. O mecanismo desta reação envolve a conversão inicial da hidroxila da oxima em um bom grupo abandonador através de sua protonação. Em seguida, o grupo alquila anti à oxima migra do carbono para o nitrogênio, através de um processo concertado. Nesta etapa, uma molécula de água é liberada e o intermediário nitrilo é formado. $O$ íon nitrilo sofre ataque nucleofílico da água e, por fim, o produto sofre tautomerização para a amida correspondente, ${ }^{39,76}$ Esquema 12.

Um exemplo do uso de oximas na Síntese Orgânica Industrial é a produção da caprolactama, um precursor do polímero policaprolactama, o Nylon 6 . Neste procedimento a cicloexanona é utilizada como material de partida para obtenção da oxima que, em seguida, sofre o Rearranjo de Beckmann em meio ácido para gerar a lactama cíclica, ${ }^{71}$ Esquema 13.<smiles></smiles>

Rearranjo de Beckmann

$X$ - ácido de Lewis

Esquema 12. Preparação de amidas através do Rearranjo de Beckmann de oximas 


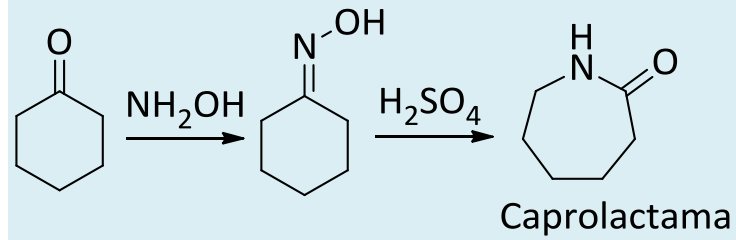

Esquema 13. Síntese do caprolactama via Rearranjo de Beckmann. Adaptado de Wittcoff e Colaboradores $(2004)^{71}$

Hill e Chortyk ao submeter a 9-acetil-cisdecalinoxima ao Rearranjo de Beckmann em presença de ácido sulfúrico observaram a formação do $\mathrm{N}$-decahidronaftaleno acetamida. Considerando que a referida amida não poderia ser gerada através do mecanismo sugerido para o Rearranjo de Beckmann, outra proposta para a reação foi construída. Nessa situação, a reação ocorreria através da fragmentação inicial da oxima e, consequente, geração de três espécies: um carbocátion estável, uma nitrila e água. Em seguida, os fragmentos se recombinam de acordo com a Figura 18 para formar a amida (Esquema 14). ${ }^{77}$

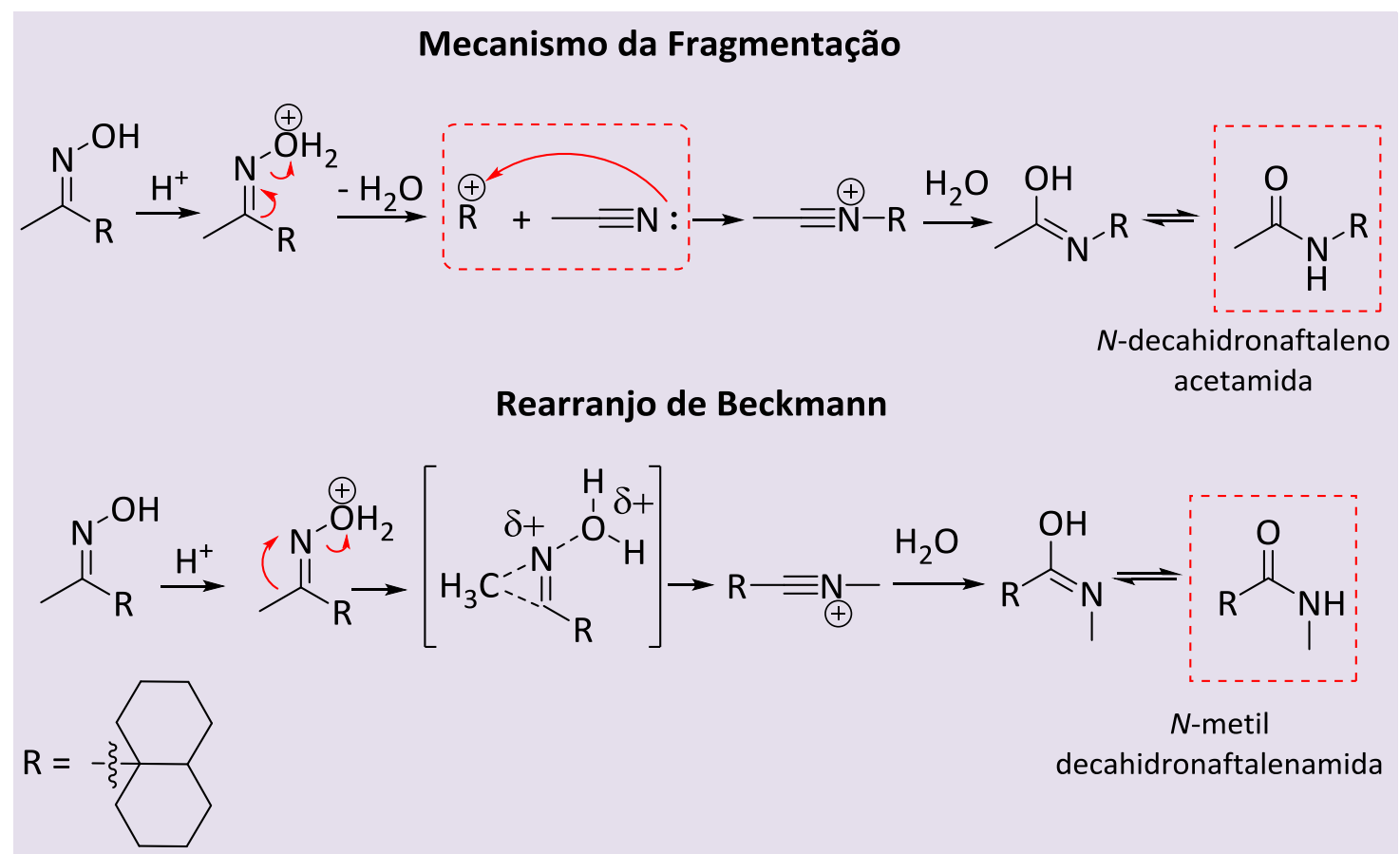

Esquema 14. Preparação de amidas a partir de oximas via intermediário carbocátion e via Rearranjo de Beckmann

4.2. Obtenção de anéis isozaxolínicos empregando oximas

As oximas podem gerar nitronas e óxidos de nitrila, dois tipos de dipolo-1,3, Figura 20. Essas espécies são preparadas a partir, respectivamente, da alquilação do nitrogênio de oximas e por desidrohalogenação de $\alpha$ haloximas. ${ }^{78}$ As nitronas e óxidos de nitrila participam de reações de adição nucleofílica, sendo assim, empregados na síntese de várias moléculas de origem natural e biologicamente ativas. Dentre estas reações, a cicloadição 1,3 com alcenos é uma das mais importantes, visto que geram anéis 
isozaxolínicos de forma regiosseletiva e estereoespecífica, ${ }^{40}$ Figura 19.

Os anéis isozaxolínicos são utilizados na síntese de várias substâncias que apresentam relevante atividade biológica, tais como: alcaloides, antipirético, bactericida, analgésico, nucleosídeo, antibióticos, $\beta$ lactamas, entre outros. ${ }^{72,73}$ Além disso, estes heteropentaciclos vêm apresentando aplicações tecnológicas nos últimos anos, visto que estão presentes em moléculas orgânicas sensíveis a efeitos externos, os chamados cristais líquidos. ${ }^{74,75}$

Nas reações de cicloadição com dipolo-1,3 (heterodienófilos), ânions do tipo alil ou propargil/alenil, reagem com o sistema $\pi$ (dipolarófilo) através de um processo concertado que envolve um estado de transição cíclico. Seis elétrons estão envolvidos no processo, sendo quatro elétrons distribuídos em três átomos (o dipolo-1,3) e dois elétrons distribuídos em dois átomos (o alceno) assim, trata-se de uma cicloadição [3+2], ${ }^{79}$ Esquema 15.

Além dos óxidos de nitrila e nitronas, ilídeos de azometano, ilídeos de carbonila, diazoalcanos e outros também são capazes de sofrer ressonância e originar dipolos do tipo $1,3,^{73}$ Esquema 16.

\section{Dipolo 1,3 do tipo propargil/alenil}

\section{Heterodienófilo}

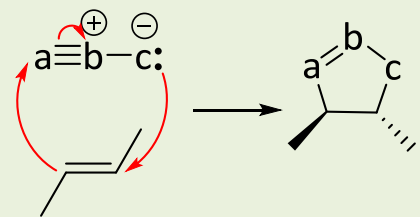

Dipolarófilo Anel Isoxazolina

Esquema 15. Reação de cicloadição 1,3-dipolar. Adaptado de Gothelf $(2001)^{79}$

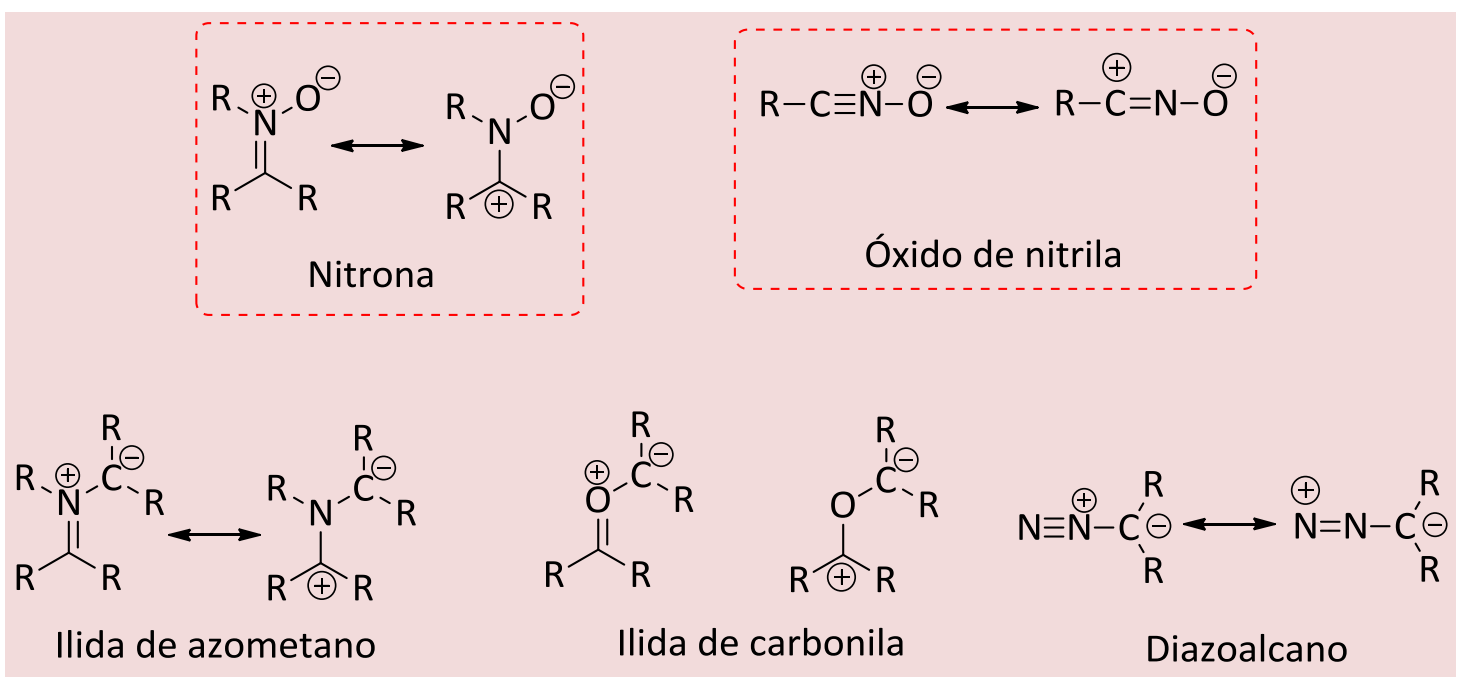

Esquema 16. Alguns tipos de dipolo 1,3. Adaptado de Melo e Colaboradores (2006) ${ }^{73}$ 
Quando alcenos 1,2-dissubstituídos estão envolvidos nas cicloadições 1,3-dipolares, dois centros quirais são formados de forma estereoespecífica. As cicloadições têm seus estados de transição controlados pelos orbitais moleculares de fronteira (OMF) dos substratos: o orbital molecular preenchido de maior energia (HOMO) e o orbital molecular vazio de menor energia (LUMO). De acordo com o tipo de interação que rege as cicloadições, estas são classificadas em três tipos: Tipo I - reação controlada pela interação entre o HOMO do dipolo e o LUMO do dipolarófilo; Tipo II - reação controlada pela interação entre o HOMO do dipolo e o LUMO do dipolarófilo, bem como, a interação entre o HOMO do dipolarófilo e o LUMO do dipolo; e Tipo III - reação controlada pela interação entre o HOMO do dipolarófilo e o LUMO do dipolo. A interação predominante será aquela onde a diferença de energia entre o HOMO e o LUMO seja menor, ${ }^{79,80}$ Figura 5.
Interação Tipo |

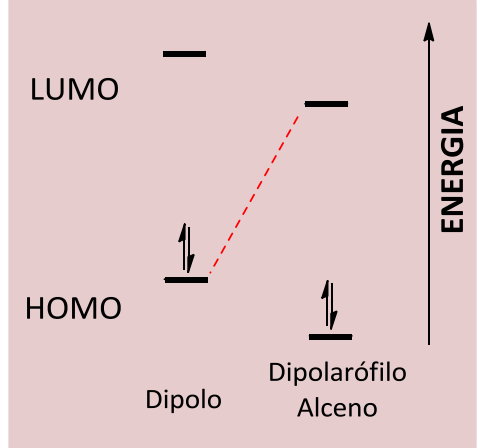

Interação Tipo II

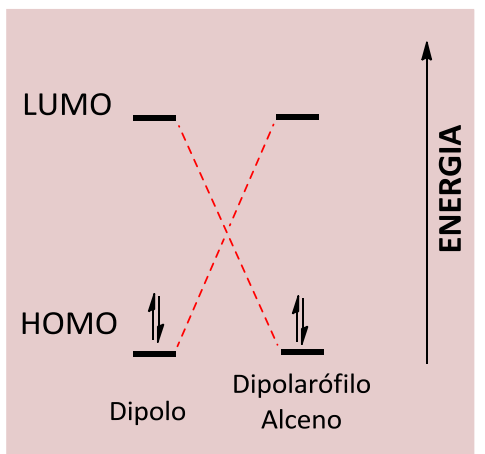

Interação Tipo III

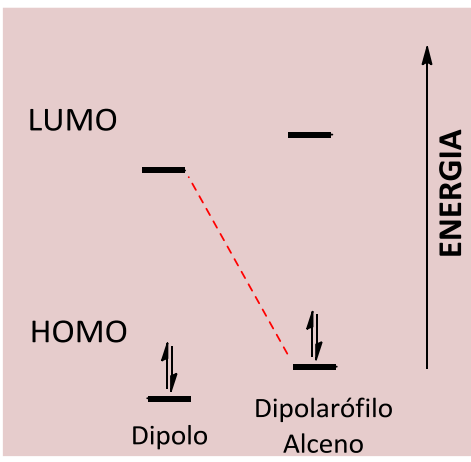

Figura 5. Esquema dos tipos de interações dos orbitais de fronteira nas reações de cicloadição 1,3-dipolar. Adaptado de Gothelf (2001) ${ }^{79}$

As nitronas foram descobertas por Beckman em 1880, mas somente após aproximadamente 40 anos é que foram utilizadas pela primeira vez em uma reação de cicloadição 1,3-dipolar, o que resultou na preparação de isoxazolidinas. Ao reagirem com alcinos e alcenos são geradas, respectivamente, isoxazolidinas e 4isozaxolinas, podendo a primeira apresentar até três centros assimétricos com estereoquímica controlada, ${ }^{81}$ Esquema 17.

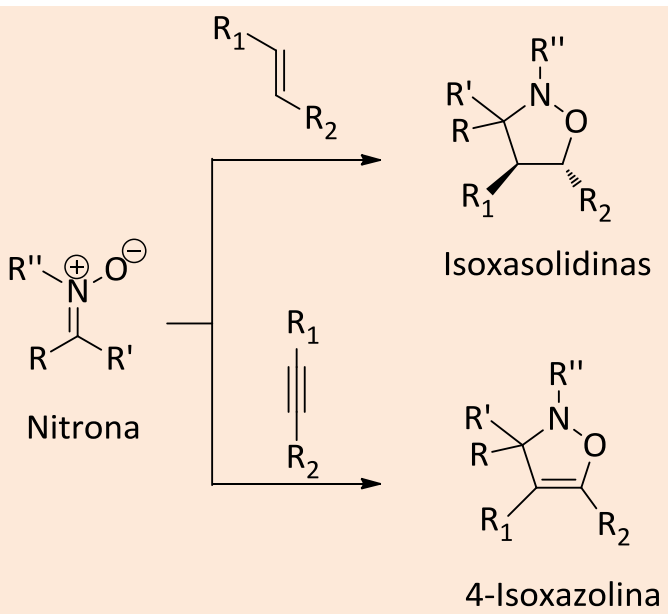

Esquema 17. Cicloadição 1,3-dipolar de nitronas com alcenos e alcinos 
Alcenos e alcinos são excelentes dipolarófilos nas reações de cicloadiação 1,3 com óxidos de nitrila, os quais podem produzir isoxazolinas e isoxazóis, respectivamente. Os dipolos 1,3, em geral, são gerados in situ em razão de sua alta reatividade e capacidade de dimerização, ${ }^{78,82}$ Esquema 18.

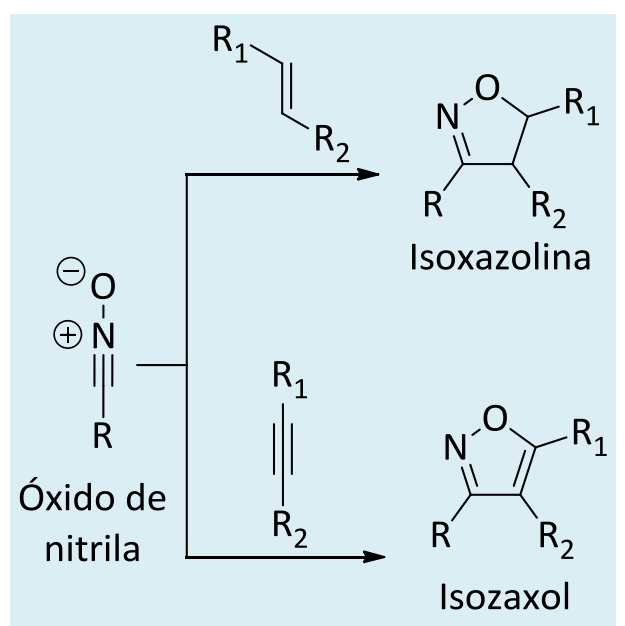

Esquema 18. Cicloadição 1,3-dipolar de óxidos de nitrila com alcenos e alcinos

Reações de cicloadição 1,3-dipolar ocorrem com alta regiosseletividade, sendo sua regioquímica influenciada por fatores estéricos e eletrônicos. Em alcenos terminais, o impedimento estérico leva à formação do isômero 3,5-dissubstituído e, neste caso, a interação dos OMF é do Tipo III. Contudo, quando o alceno terminal possui como substituinte um grupo elétron-retirador (GER) tanto o isômero 3,5-GER-substituído quanto o 3,4-GER-dissubstituído são produzidos. Neste caso, a regioquímica da reação também estará sofrendo a influência do efeito eletrônico, visto que o GER reduz a energia do LUMO do alceno e agora a reação será conduzida pela interação dos OMF do Tipo I. Nos casos em que o alceno é dissubstituído o efeito estérico é eliminado e apenas o isômero 3,4-GER-dissubstituído é formado, ${ }^{79,80}$ Esquema 19.

Além de originar heteropentaciclos, os óxidos de nitrila podem formar 1,2,5oxadiazol 4-óxidos, 1,2,4-oxadiazol 4-óxidos e 1,4,2,5-dioxadiazinas. ${ }^{81}$ 


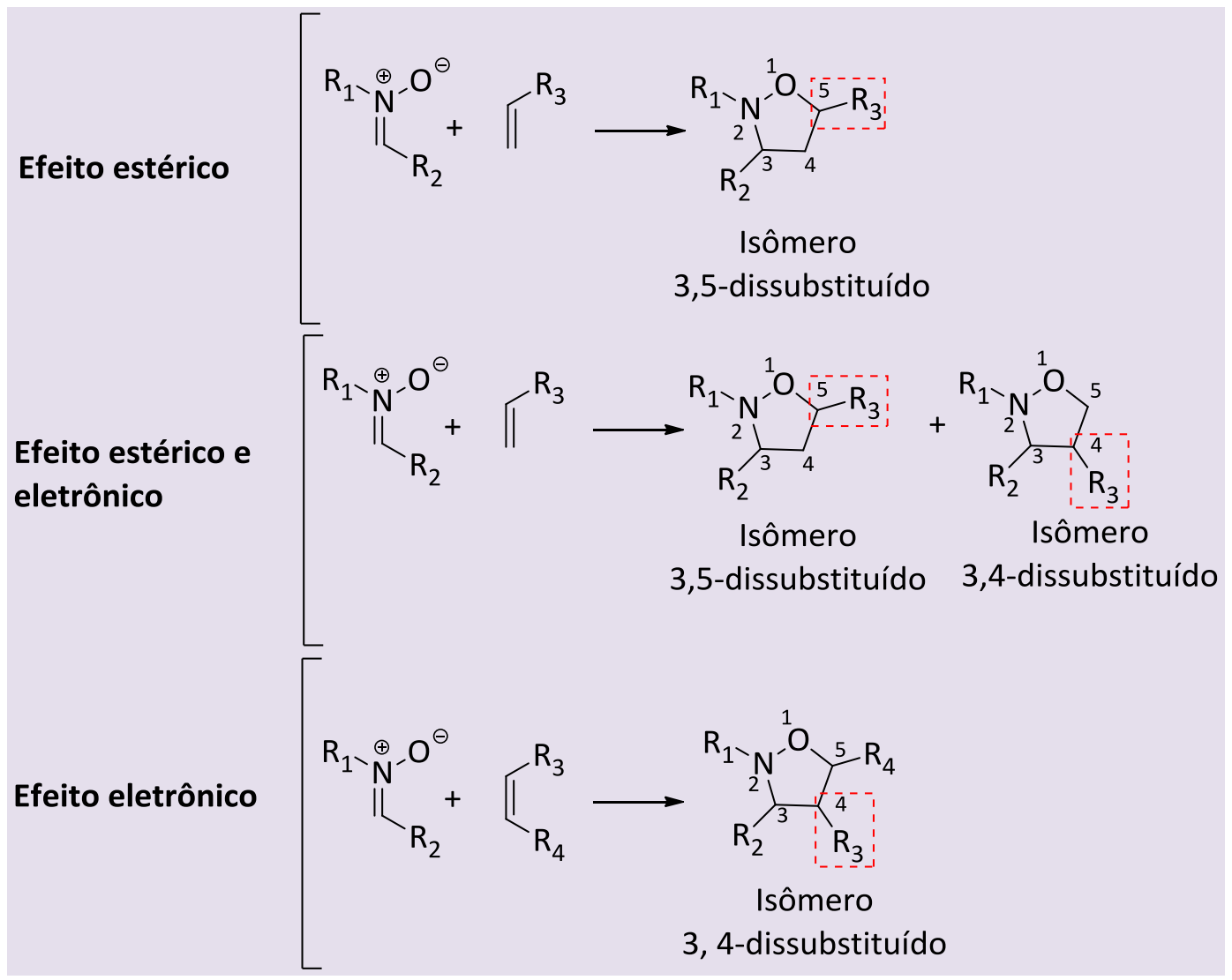

Esquema 19. Regiosseletividade da reação de cicloadição 1,3-dipolar

4.3. Obtenção de nitrosos a partir de oximas

Compostos gem-cloro nitrosos são obtidos a partir da reação de aldoximas ou cetoximas com cloreto de nitrosila. Kyung e Clapp mostraram que apenas algumas oximas produzem gem-cloro nitrosos quando tratados com NOCl, outras formam nitroiminas (compostos pernitrosos) ou cetonas, isso, a depender das condições reacionais, como: polaridade do solvente e concentrações de $\mathrm{NOCl}$ e oxima. ${ }^{41} \mathrm{O}$ principal intermediário desta reação é a nitrosonitrona, formada após a reação do $\mathrm{NO}^{+}$com a oxima. Em seguida, esta sofre um ataque nucelofílico do cloreto para a geração do gem-cloro nitroso, Figura 25. A proposta mecanística para a produção da nitroimina e da cetona envolve um rearranjo da nitrosanitrona, passando por um anel de três membros, ${ }^{41,55,83}$ conforme Esquema 20.

A metodologia mais utilizada para obter compostos gem-halo nitrosos é a partir da reação entre a oxima e o halogênio ou hipohaleto de alquila. Este método foi utilizado por vários grupos em 1950. Diekman e Luttke prepararam alguns compostos gemcloro nitrosos ( $\mathrm{RR}^{\prime} \mathrm{ClCN}=\mathrm{O} ; \mathrm{R} / \mathrm{R}^{\prime}=\mathrm{H} / \mathrm{Me}$; $\mathrm{H} / \mathrm{Ph}$; $\mathrm{Me} / \mathrm{Me}$; $\mathrm{Me} / t-\mathrm{Bu} ; \quad \mathrm{Me} / n-\mathrm{Bu}$; $\mathrm{Me} / \mathrm{CH}_{2} \mathrm{Ph}$ ) com altos rendimentos (>90\%) empregando hipoclorito de terc-butila. ${ }^{84}$ 


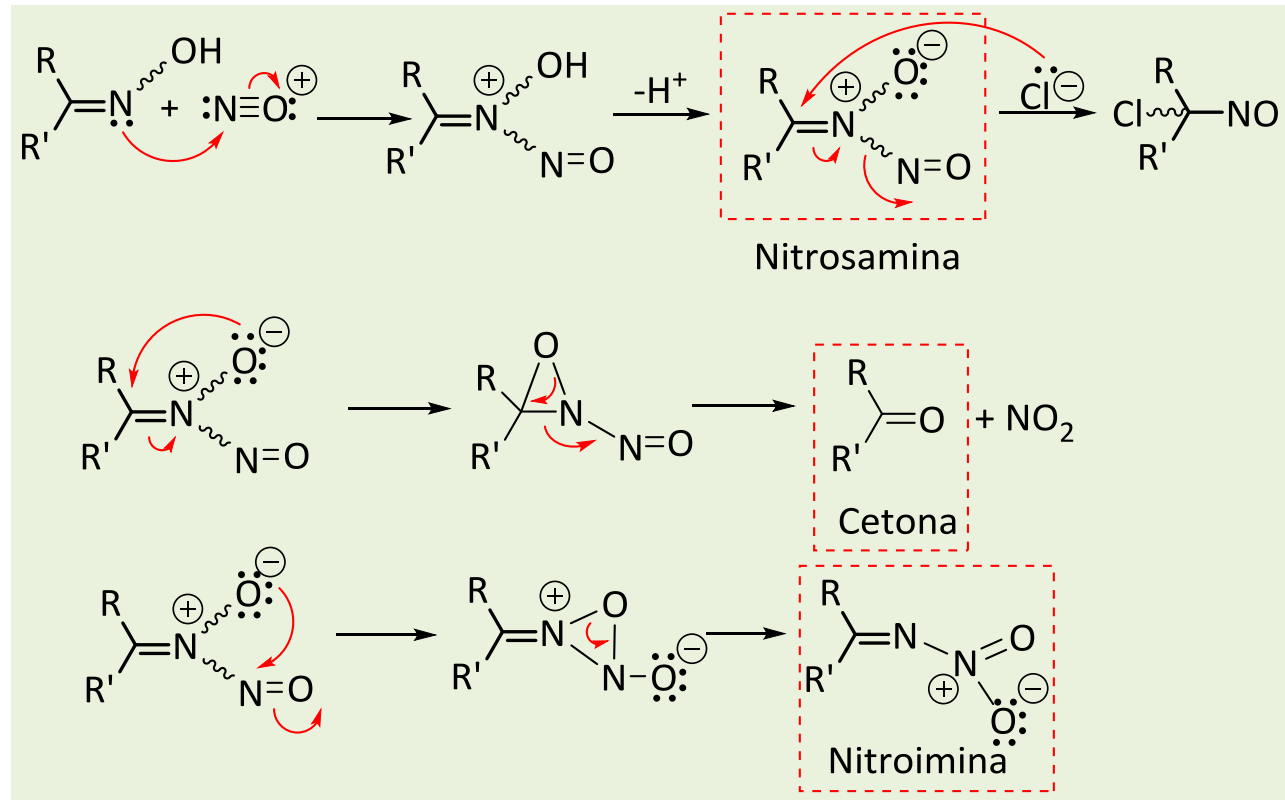

Esquema 20. Obtenção de compostos gem-cloro nitrosos a partir de oximas. Adaptado de Kyung e Clapp (1976) ${ }^{41}$

Há vários trabalhos que apresentam a preparação de vinil nitrosos a partir da desidrohalogenação de $\alpha$-haloximas utilizando bases. Muitos dos nitrosoalcenos formados são altamente reativos e têm apenas uma existência transitória à temperatura ambiente, contudo, existem outros que são sólidos estáveis. Dentre as bases utilizadas na desidrohalogenação de cloroximas estão a trietilamina, o 1,5diazobiciclo $[4,3,0]$ non-5-eno, ${ }^{85}$ os alcoóxidos de sódio, a suspensão de carbonato de sódio em $t$-butilmetil éter, a mistura de carbonato de sódio em diclorometano, ${ }^{86,87}$ o bicarbonato de sódio em álcool e o hidróxido de cálcio em suspensão de acetato de etila, ${ }^{88}$ Esquema 21.

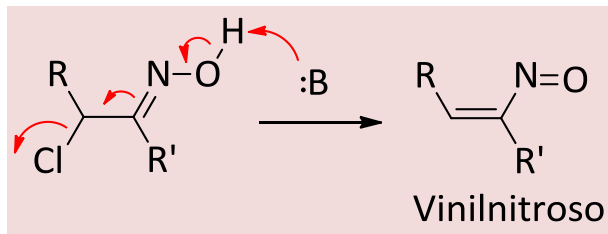

Esquema 21. Obtenção de vinilnitrosos a partir de $\alpha$-cloroximas

4.4. Obtenção de nitrocompostos através da oxidação de oximas

Os nitrocompostos podem ser preparados a partir da oxidação de oximas. Nessa metodologia são empregados agentes fortes de oxidação, tal como o ácido peroxitrifluoroacético $\left(\mathrm{CF}_{3} \mathrm{CO}_{3} \mathrm{H}\right)$. Várias condições reacionais para a preparação de nitrocompostos a partir de oximas foram desenvolvidas e algumas estão mostradas na Tabela 2. 
Tabela 2. Condições reacionais para a oxidação de oximas para nitrocompostos

\begin{tabular}{|c|c|c|}
\hline \multicolumn{3}{|c|}{ PREPARAÇÃO DE NITROCOMPOSTOS A PARTIR DE OXIMAS } \\
\hline $\mathbf{R}, \mathbf{R}^{\prime}$ & Condições reacionais & Referências \\
\hline $\mathrm{R}=\mathrm{O}^{\bar{\nu}} \mathrm{O} \mathrm{O} \mathrm{R}^{\prime}=\mathrm{H}$ & $\mathrm{PUH} \bullet\left(\mathrm{CF}_{3} \mathrm{CO}\right)_{2} \mathrm{O}, \mathrm{CH}_{3} \mathrm{CN}, 0^{\circ} \mathrm{C}$ & 2 \\
\hline $\mathrm{R}=-\xi-\mathrm{OMe} \quad \mathrm{R}^{\prime}=\mathrm{H}$ & $\mathrm{PUH} \bullet\left(\mathrm{CF}_{3} \mathrm{CO}\right)_{2} \mathrm{O}, \mathrm{CH}_{3} \mathrm{CN}, 0^{\circ} \mathrm{C}$ & 2 \\
\hline 2-Oxima bicicloheptano & $\mathrm{NaBO}_{3} \bullet \mathrm{H}_{2} \mathrm{O}, \mathrm{AcOH}, 55-65^{\circ} \mathrm{C}$ & 89 \\
\hline 1-Oxima ciclododecano & $\mathrm{BzOMo}\left(\mathrm{O}_{2}\right)_{2}, \mathrm{CH}_{3} \mathrm{CN}, 40^{\circ} \mathrm{C}$ & 90 \\
\hline $\mathrm{R}=\mathrm{s}^{\mathrm{s}} \mathrm{R}^{\prime}=\mathrm{H}$ & $\mathrm{BzOMo}\left(\mathrm{O}_{2}\right)_{2}, \mathrm{CH}_{3} \mathrm{CN}, 40^{\circ} \mathrm{C}$ & 90 \\
\hline 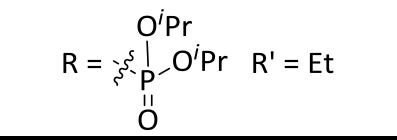 & $m$-CPBA, $\mathrm{CH}_{2} \mathrm{Cl}_{2}$, T.A. & 91 \\
\hline
\end{tabular}

PHU - complexo de peróxido de hidrogênio e ureia; $m$-CPBA - ácido meta-cloroperbenzoico

Na oxidação de oximas a nitrocompostos, os halonitrocompostos podem ser intermediários da reação. Inicialmente a oxima é oxidada para compostos halonitrosos. Nessa etapa, vários agentes podem ser empregados, tais como: cloro, bromo, solução aquosa de ácido hipocloroso, hipoclorito terc-butílico, $\quad \mathrm{N}$ bromossuccinimida (NBS). O halo nitroso formado é oxidado para halo nitrocomposto utilizando ácido nítrico, ozônio, solução aquosa de hipoclorito de sódio ou hipoclorito de $n$-butilamônio. A conversão de oximas para cloronitrocomposto pode ser realizada em etapa única. Por exemplo, o cloronitrociclohexano foi obtido com $98 \%$ de rendimento após tratar a oxima do ciclohexano com ácido hipocloroso e, em seguida, com uma mistura de hidrogenossulfato de tetra-n-butilamônio e hipoclorito de sódio. A decloração redutiva do? ?cloronitrocomposto é realizada através de hidrogenólise catalítica com $\mathrm{H}_{2}$ e $\mathrm{Pd} / \mathrm{C}$ em metanol-água, ${ }^{42,92}$ Esquema 22.

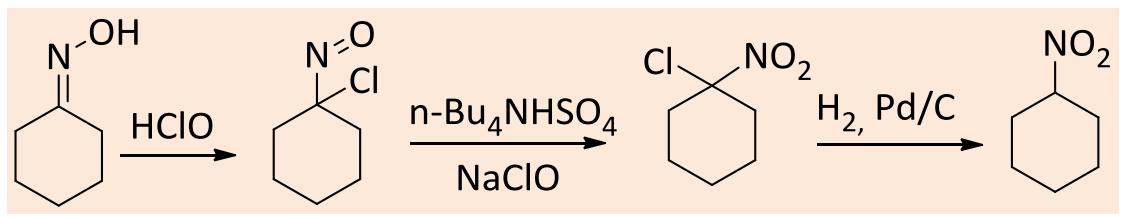

Esquema 22. Oxidação da oxima do ciclohexano para 1-nitrocicloexano

4.5. Obtenção de nitrilas através da desidratação de oximas

A desidratação de aldoximas é uma metodologia bastante utilizada para a preparação de nitrilas. Diversos trabalhos estudaram esta reação e, como consequência, existem, atualmente, várias condições reacionais onde as nitrilas são preparadas a partir de aldoximas, algumas destas estão mostradas na Tabela 3. Cabe salientar que cetoximas também podem ser convertidas em nitrilas em presença de ácidos de Lewis. ${ }^{43}$ 
Tabela 3. Condições reacionais para a desidratação de oximas para nitrilas

\section{PREPARAÇÃO DE NITRILAS A PARTIR DE ALDOXIMAS}

\begin{tabular}{|c|c|c|}
\hline $\mathbf{R}$ & Condições reacionais & Referências \\
\hline$n-\mathrm{Bu}, \mathrm{Me}, \mathrm{Ph}$ & $(\mathrm{OR})_{2} \mathrm{HP}=\mathrm{O}, \mathrm{Et}_{3} \mathrm{~N}, \mathrm{CCl}_{4}$ & 93 \\
\hline $\mathrm{Ph}$ & $\left(\mathrm{HNSiMe}_{2}\right)_{3}$ & 94 \\
\hline$n-\mathrm{C}_{7} \mathrm{H}_{15}, \mathrm{Ph}, p-\mathrm{ClC}_{6} \mathrm{H}_{4}, \mathrm{PhCH}=\mathrm{CH}$ & 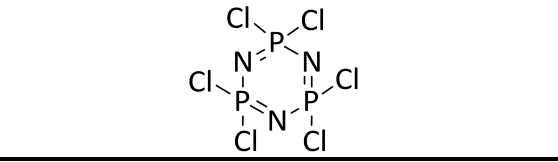 & 95 \\
\hline $\begin{array}{c}\mathrm{Pr}, n-\mathrm{C}_{7} \mathrm{H}_{5}, p-\mathrm{NO}_{2} \mathrm{C}_{6} \mathrm{H}_{4} \\
p-\mathrm{ClC}_{6} \mathrm{H}_{4}, \mathrm{PhCHCH} \\
\end{array}$ & $\mathrm{P}_{2} \mathrm{I}_{4}$ & 96 \\
\hline $\mathrm{Ph}$ & $\begin{array}{c}\mathrm{KCN},\left(\mathrm{CH}_{3}\right)_{3} \mathrm{SiCl}, 18 \text {-crown éter-6, } \\
\mathrm{CH}_{3} \mathrm{CN}\end{array}$ & 97 \\
\hline $\begin{array}{c}\mathrm{Pr}, \mathrm{C}_{5} \mathrm{H}_{11}, \mathrm{Ph}, p-\mathrm{ClC}_{6} \mathrm{H}_{4} \\
o-\mathrm{ClC}_{6} \mathrm{H}_{4}, p-\mathrm{NO}_{2} \mathrm{C}_{6} \mathrm{H}_{4} \\
\end{array}$ & $\mathrm{~N}^{-} \mathrm{N}-\mathrm{COCF}_{3}$ & 98 \\
\hline$c-\mathrm{C}_{6} \mathrm{H}_{11}, \mathrm{Ph}, p-\mathrm{NO}_{2} \mathrm{C}_{6} \mathrm{H}_{4}, \mathrm{PhCHCH}$ & & 99 \\
\hline $\mathrm{Et}, n-\mathrm{Bu}, c-\mathrm{C}_{6} \mathrm{H}_{11}, \mathrm{Ph}$ & $\mathrm{Rh}_{6}(\mathrm{CO})_{16}, \mathrm{CO}, \mathrm{H}_{2} \mathrm{O}$ & 100 \\
\hline $\begin{array}{c}\mathrm{Ph}, p-\mathrm{BrC}_{6} \mathrm{H}_{4}, o-\mathrm{OMeC}_{6} \mathrm{H}_{4} \\
p-\mathrm{NO}_{2} \mathrm{C}_{6} \mathrm{H}_{5}, p-\mathrm{OHC}_{6} \mathrm{H}_{5}\end{array}$ & $\begin{array}{c}{\left[\mathrm{RuCl}_{2}(p \text {-cimeno })\right], \text { peneira }} \\
\text { molecular }\end{array}$ & 101 \\
\hline $\begin{array}{c}\mathrm{Pr}, o-\mathrm{OMeC}_{6} \mathrm{H}_{4}, p-\mathrm{OMeC}_{6} \mathrm{H}_{5}, \\
m-\mathrm{NO}_{2} \mathrm{C}_{6} \mathrm{H}_{4}, p-\mathrm{MeC}_{6} \mathrm{H}_{4} \\
o-\mathrm{OHC}_{6} \mathrm{H}_{4}, p-\mathrm{OHC}_{6} \mathrm{H}_{4}, m-\mathrm{ClC}_{6} \mathrm{H}_{4} \\
\text { l }_{\mathrm{S}}\end{array}$ & $\mathrm{Al}_{2} \mathrm{O}_{3}, \mathrm{MeSO}_{2} \mathrm{Cl}$ & 102 \\
\hline $\begin{array}{c}n-\mathrm{C}_{7} \mathrm{H}_{15}, \mathrm{Ph}, p-\mathrm{OMeC}{ }_{6} \mathrm{H}_{4} \\
p-\mathrm{MeC}_{6} \mathrm{H}_{4}, \mathrm{PhCH}=\mathrm{CH}, p-\mathrm{ClC}_{6} \mathrm{H}_{4}\end{array}$ & $\mathrm{AlCl}_{3} \cdot 6 \mathrm{H}_{2} \mathrm{O} / \mathrm{KI} / \mathrm{H}_{2} \mathrm{O} / \mathrm{CH}_{3} \mathrm{CN}$ & 103 \\
\hline $\begin{array}{c}\mathrm{Ph}, p-\mathrm{OMeC}_{6} \mathrm{H}_{4} \\
\text { 1-nafitil, } \mathrm{PhCH}=\mathrm{CH}\end{array}$ & $\mathrm{ClSO}_{2} \mathrm{~F}, \mathrm{Et}_{3} \mathrm{~N}$ & 104 \\
\hline $\begin{array}{l}n-\mathrm{C}_{7} \mathrm{H}_{15}, n-\mathrm{C}_{9} \mathrm{H}_{19}, \mathrm{Ph} \\
p-\mathrm{NO}_{2} \mathrm{C}_{6} \mathrm{H}_{4}, \mathrm{PhCH}_{2} \mathrm{CH}_{2}\end{array}$ & $\mathrm{Cu}(\mathrm{OAc})_{2}$ & 105 \\
\hline $\mathrm{Ph}$ & $\mathrm{All}_{3}, \mathrm{MeCN}$ & 106 \\
\hline $\begin{array}{c}\mathrm{Ph}, p-\mathrm{OMeC}_{6} \mathrm{H}_{4}, o-\mathrm{OHC}_{6} \mathrm{H}_{4} \\
p-\mathrm{OHC}_{6} \mathrm{H}_{4}, o-\mathrm{NMe}_{2} \mathrm{C}_{6} \mathrm{H}_{4} \\
\end{array}$ & $\mathrm{SiO}_{2}-\mathrm{OPOCl}_{2}$ & 107 \\
\hline $\begin{array}{c}\mathrm{Pr}, p-\mathrm{ClC}_{6} \mathrm{H}_{4}, n-\mathrm{C}_{7} \mathrm{H}_{5} \\
p-\mathrm{OMeC}_{6} \mathrm{H}_{4}, p-\mathrm{NO}_{2} \mathrm{C}_{6} \mathrm{H}_{4}\end{array}$ & 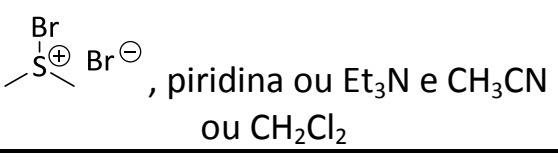 & 108 \\
\hline $\begin{array}{c}\mathrm{Ph}, o-\mathrm{OMeC}_{6} \mathrm{H}_{4}, p-\mathrm{OMeC}_{6} \mathrm{H}_{4} \\
p-\mathrm{CF}_{3} \mathrm{OC}_{6} \mathrm{H}_{4}, o-\mathrm{ClC}_{6} \mathrm{H}_{4}, o-\mathrm{NO}_{2} \mathrm{C}_{6} \mathrm{H}_{4} \\
p-\mathrm{NO}_{2} \mathrm{C}_{6} \mathrm{H}_{4}, o-\mathrm{OHC}_{6} \mathrm{H}_{4}\end{array}$ & 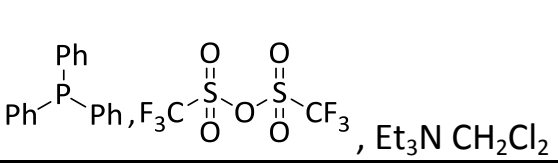 & 109 \\
\hline
\end{tabular}


4.6. Obtenção de aminas através da redução de oximas

Aminas podem ser preparadas a partir da redução de cetoximas e aldoximas empregando-se vários agentes redutores, tais como: sódio metálico, ${ }^{44} \mathrm{LiAlH}_{4}{ }^{110} \mathrm{NaH}$, formiato de amônio/Pd/C, formiato de amônio /magnésio, ${ }^{111} \mathrm{H}_{2}$ e Raney Nickel, ${ }^{112}$ 1,3,2-oxazaborolidinas, ésteres espiroboranos, ${ }^{113}$ boranos $\left(\mathrm{BH}_{3} \bullet \mathrm{THF}\right),{ }^{114}$ $\mathrm{RuCl}_{2}\left(\mathrm{PPh}_{3}\right),{ }^{115} \mathrm{NaBH}_{4} / \mathrm{TiCl}_{4},{ }^{116}$ Esquema 23.

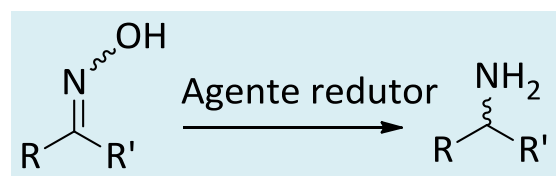

Esquema 23. Obtenção de aminas a partir da redução de oximas

\section{Considerações Finais}

O grupo oxima tem importância inquestionável para a química de coordenação a para a Síntese Orgânica, contudo há uma carência do tema nos livros em língua portuguesa adotados nos cursos de ensino superior do Brasil. Assim, o presente trabalho reuniu as principais informações sobre as propriedades químicas de oximas, as metodologias de obtenção e alguns empregos desta classe de compostos na Síntese Orgânica. Desta forma, estudantes de graduação, pós-graduação e pesquisadores interessados pelo tema podem utilizar esta revisão para auxiliar seus estudos.

\section{Referências Bibliográficas}

${ }^{1}$ Chaudhuri, P. Homo- and heteropolymetallic exchange coupled metaloximates. Coordination Chemistry Reviews 2003, 243, 143. [CrossRef]

${ }^{2}$ Kukushkin, V. Y.; Tudela, D.; Pombeiro, A. J. L. Metal-ion assisted reactions of oxime and reactivity of oxime-containing metal complexes. Coordination Chemistry Reviews 1996, 156, 333. [CrossRef]

${ }^{3}$ Smith, A. G.; Tasker, P. A.; White, D. J. The structures of phenolic oximes and their complexes. Coordination Chemistry Reviews 2003, 241, 61. [CrossRef]
${ }^{4}$ Sítio dos Periódicos Capes. Disponível em: <www.periodicos.capes.gov.br/>. Acesso em: 1 dezembro 2014.

${ }^{5}$ Miyata, O.; Hashimoto, J.; Iba, R.; Naito, T. Preparation of optically active ?-hydroxy oxime ether bydiastereoselective imino 1,2Wittig rearrangement of hydroximates and its application to synthesis of (+)-cytoxazone. Tetrahedron Letters 2005, 46, 4015. [CrossRef]

${ }^{6}$ Arena, J. M. Poisoning: Toxicology, Symptoms, Treatments, 4a. ed., Charles C. Tomas: Springfield, 1979.

${ }^{7}$ Hoffman, R. V. Em Organic Chemistry. An Intermediate Text, 2a. ed., Wiley: New Jersey, 2004, cap 7.

${ }^{8}$ Dieter, R. K.; Datar, R. 1,2-Nucleophilic additions of organolithium reagents to chiral oxime ethers. Canadian Journal of Chemistry 1993, 71, 814. [CrossRef]

${ }^{9}$ Dindar, M. H.; Fathi, S. A. M.; Yaftian, M. R.; Noushiranzadeh, N. Solid phase extraction of copper(II) ions using C18-silica disks modified by oxime ligands. Journal of Hazardous Materials 2010, 179, 289. [CrossRef] [Pubmed]

${ }^{10}$ Corsaro, A.; Chiacchio, M. A.; Pistarà, V. Regeneration of Carbonyl Compounds from the Corresponding Oximes: An Update Until to 2008. Current Organic Chemistry 2009, 13, 482. [CrossRef]

${ }^{11}$ Vollhardt, K. P. C.; Schore, N. E. Em Química Orgânica. Estrutura e Função, 4ạ. ed., Bookman: Porto Alegre, 2004, cap. 17. 
${ }^{12}$ Milios, C. J.; Stamatatos, T. C.; Perlepes, S. $P$. The coordination chemistry of pyridyl oximes. Polyhedron 2006, 25, 134. [CrossRef]

${ }^{13}$ Nsikabaka, S.; Harb, W.; Ruiz-López, M. F. The role of water on the acid-promoted $E / Z$ isomerization of oximes in aqueous solution. Journal of Molecular Structure: THEOCHEM 2006, 764, 161. [CrossRef]

${ }^{14}$ Sakaizumi, T.; Sasane, I.; Kouno, T.; Takeda, S.; Kuze, N.; Ohashi, O.; lijima, K. Microwave spectrum, molecular structure, and ab initio calculation of $(E)$ chloroacetaldehyde oxime. Journal of Molecular Structure 1997, 413-414, 107. [CrossRef]

${ }^{15}$ lijima, K.; Suzuki, M.; Sakaizumi, T.; Ohashi, $\mathrm{O}$. Molecular structure of gaseous acetoxime determined by electron diffraction. Journal of Molecular Structure 1997, 413-414, 327. [CrossRef]

${ }^{16}$ Golec, B.; Grzegorzek, J.; Mielke, Z. Complexation of formaldoxime and acetaldoxime with nitrogen. Chemical Physics 2008, 353, 13. [CrossRef]

${ }^{17}$ Ciunik, Z. Configurational disorder in oximes: low temperature $\mathrm{X}$-ray single crystal investigations. Journal of Molecular Structure 1996, 385, 89. [CrossRef]

${ }^{18}$ Clayden, J.; Geeves, N.; Warren, S.; Wothers, P. Em Organic Chemistry, 1a. ed., Oxford University Press: New York, 2001, cap 14.

${ }^{19}$ Demetgül, C.; Serin, S. Synthesis and characterization of a new vic-dioxime derivative of chitosan and its transition metal complexes. Carbohydrate Polymers 2008, 72, 506. [CrossRef]

${ }^{20}$ Vogh, J. W. Isolation and analysis of carbonyl compound as oximes. Analytical Chemistry 1971, 43, 1618. [CrossRef]

${ }^{21}$ Worek, F.; Thiermanna, H.; Szinicza, L.; Eyer; P. Kinetic analysis of interactions between human acetylcholinesterase, structurally different organophosphorus compounds and oximes. Biochemical Pharmacology 2004, 68, 2237. [CrossRef] [PubMed]

${ }^{22}$ Tiwari, S.; Ghosh, K. K.; Marek, J.; Kuca, K. Spectrophotometric Determination of the Acidity Constants of Some Oxime-Based $\alpha$ Nucleophiles. Journal of Chemical \& Engineering Data 2010, 55, 1153. [Crossref]
${ }^{23}$ Smith, M. B.; March, J. Em March's Advanced Organic Chemistry. Reactions, mechanisms, and structure, 5a. ed., Wiley: New York, 2001, cap. 15.

${ }^{24}$ Smith, M. B.; March, J. Em March's Advanced Organic Chemistry. Reactions, mechanisms, and structure, 5a. ed., Wiley: New York, 2001, cap. 12.

${ }^{25}$ Long, J. A.; Harris, N. J.; Lammertsma, K. Formaldehyde oxime $\longleftrightarrow \rightarrow$ nitrosomethane tautomerism. The Journal of Organic Chemistry 2001, 66, 6762. [CrossRef] [PubMed]

${ }^{26}$ Mallya, M. N.; Nagendrappa, G. Nitrosyl chloride addition to cycloalkenyltrimethylsilanes. An unexpected reversal of regiochemistry of addition, and synthesis of $\beta$-aminosilanes. Arquive for Organic Chemistry 2006, 14, 155. [CrossRef]

${ }^{27} \mathrm{Di}$ Giacomo, A. Amine-catalyzed isomerization of nitrosocyclohexane to oxime. The Journal of Organic Chemistry 1965, 30, 2614. [CrossRef]

${ }^{28}$ Glaser, R.; Murmann, R. K.; Barnes, C. L. Why do nitroso compounds dimerize while their oxime tautomers do not? A structural study of the trans-dimer of 2-chloro-2methyl-3-nitrosobutane and higher level ab initio study of thermodynamic stabilities and electronic structures of isomers of diazene dioxides. The Journal of Organic Chemistry 1996, 61, 1047. [CrossRef]

${ }^{29}$ Silverstein, R. M.; Bassler, G. C.; Morrill, T. C.; Identificação Espectrométrica de Compostos Orgânicos, 2a ed., Guanabara Dois: Rio de Janeiro, 1979.

${ }^{30}$ Canpolat, E.; Yazici, A.; Kaya, M. Studies on mononuclear chelates derived from substituted Schiff-base ligands (Part 10): synthesis and characterization of a new 4 hydroxysalicyliden- $p$ -

aminoacetophenoneoxime and its complexes with $\mathrm{Co}(\mathrm{II}), \mathrm{Ni}(\mathrm{II}), \mathrm{CU}(\mathrm{II})$ and $\mathrm{Zn}(\mathrm{II})$. Journal of Coordination Chemistry 2007, 60, 473. [CrossRef]

${ }^{31}$ Forman, S. E. Synthesis of oximes. Journal of Organic Chemistry 1964, 29, 3323. [CrossRef]

${ }^{32}$ Silverstein, R. M.; Webster, F. X.; Kiemle, D. J. Em Spectrometric Identification of Organic 
Compound, 7a. ed., John While, 2005, cap. 2, 3, 4 e 5 .

${ }^{33}$ Damljanovic, I.; Vukicevic, M.; Vukicevic, R. D. A Simple synthesis of oximes. Monatshefte für Chemie 2006, 137, 301. [CrossRef]

${ }^{34}$ Gowenlock, B. G.; Richter-Addo, G. B. Preparations of $C$-nitroso compounds. Chemical Reviews 2004, 104, 3315. [CrossRef] [PubMed]

${ }^{35}$ Kahr, K.; Berther, C. Katalystische oxydation von primaren aminen zu oximen mit wasserstoffperoxyd. Chemische Berichte 1960, 93, 132. [CrossRef]

36 Johnson, K.; Degering, Ed. F. The utilization of aliphatic nitro compounds. (I) The production of amines and the production of oximes. Journal of the American Chemical Society 1939, 61, 3194. [CrossRef]

37 Barton, D. H. R.; Beaton, J. M.; Geller, L. E.; Pechet, M. M. A new photochemical reaction. Journal of the American Chemical Society 1961, 83, 4076. [CrossRef]

38 Smith, M. B.; March, J. Em March's Advanced Organic Chemistry. Reactions, mechanisms, and structure, 5a. ed., Wiley: New York, 2001, cap. 10.

39 Blatt, A. H. The Beckmann rearrangement. Chemical Reviews 1933, 12, 215. [CrossRef]

40 Grigor'ev, I. A. Em Nitrile oxides, nitrones and nitronates in organic synthesis: Novel strategies in synthesis; Feuer, H., ed.; 2a ed., John Wiley \& Sons, 2007, cap. 2.

${ }^{41}$ Kyung, J. H.; Clapp, L. B. Pathway control of products in the reaction of nitrosyl chloride on oximes. The Journal of Organic Chemistry 1976, 41, 2024. [CrossRef]

42 Ono, N. Em The nitro group in organic synthesis. 1a. ed.; Wiley - VHC, 2001, cap. 2.

${ }^{43}$ Smith, M. B.; March, J. Em March's Advanced Organic Chemistry. Reactions, mechanisms, and structure, 5a. ed., Wiley: New York, 2001, cap. 17.

${ }^{44}$ Mosher, W. A.; Cox Jr., C.; Kreitzer, J. 2,2Dimethyl-3-aminopentane and its reaction with nitrosyl chloride. Journal of the Chemical Society 1953, 75, 4605. [CrossRef]

45 Beckmann, E. Zur isomerie der aldoxime. Berichte der Deutschem chemischem Gesellschaft 1890, 23, 1680. [CrossRef]
${ }^{46}$ Beckmann, E. Zur kenntnis der oximidoverbindungen. Justus Liebigs Annalen der Chemie 1909, 365, 201. [CrossRef]

${ }^{47}$ Carey, F. A. Em Organic chemistry, 4a. ed., 2000, cap. 17.

${ }^{48}$ Smith, M. B.; March, J. Em March's Advanced Organic Chemistry. Reactions, mechanisms, and structure, 5a. ed., Wiley: New York, 2001, cap. 16.

49 Ballini, R.; Barboni, L.; Filippone, P. Amberlyst A-21 an excellent heterogeneous catalyst for the conversion of carbonyl compounds to oximes. Chemistry Letter 1997, 26, 475. [CrossRef]

${ }^{50}$ Saikia, L.; Baruah, J. M.; Thakur, A. J. A rapid, convenient, solventless green approach for the synthesis of oximes using grindstone chemistry. Organic and Medicinal Chemistry Letters 2011, 1, 12. [CrossRef] [PubMed]

51 Tilden, W. A.; Shenstone, W. A. XIXIsomeric nitroso-terpenes. Journal of the Chemical Society 1877, 31, 554. [CrossRef]

52 Tilden, W. A. XXIV-On the action of nitrosyl chloride on organic bodies. PART II. On Turpentine Oil. Journal of the Chemical Society 1875, 28, 514. [CrossRef]

53 Meinwaldy, J.; Meinwaladn, V. C.; Aker, D. T. N. The addition of nitrosyl halides to olefins. Dependence of steric course on olefin structure. Journal of the American Chemical Society 1964, 86, 4074. [CrossRef]

54 Ponder, B. W.; Walker, D. R. Addition of nitrosyl chloride to olefins. Synthesis of some chloro ketones. Journal of the American Chemical Society 1967, 32, 4136. [CrossRef]

${ }^{55}$ Beckham, L. J.; Fessler, W. A.; Kise, M. A. Nitrosyl chloride. Chemical Reviews 1951, 48, 319. [CrossRef] [PubMed]

56 Thorne, N. 2-Methylbut-2-ene nitrosochloride and its derivatives. Journal of the Chemical Society 1956, 2587. [CrossRef]

57 Thorne, N. The formation of nitrosochlorides and nitrosates. Journal of the Chemical Society 1956, 4271. [CrossRef]

${ }^{58}$ Holmes, R. R.; Bayer, R. P. A simple method for the direct oxidation of aromatic amines to nitroso compounds. Journal of the American Chemical Society 1960, 82, 3454. [CrossRef] 
${ }^{59}$ Zajac, W. W.; Darcy Jr., M. G.; Subong, A. P.; Buzby, J. H. Oxidation of primary aliphatic amines with sodium perborate. Tetrahedron Letters 1989, 30, 6495. [CrossRef]

${ }^{60}$ Corey, E. J.; Gross, A. W. Tert-butyl-tertoctylamine. Organic Synthesis 1987, 65, 166. [CrossRef]

${ }^{61}$ Smith, M. B.; March, J. Em March's Advanced Organic Chemistry. Reactions, mechanisms, and structure, 5a. ed., Wiley: New York, 2001, cap. 19.

${ }^{62}$ Emmons, W. D. The synthesis of nitrosoalkane dimers. Journal of the American Chemical Society 1957, 79, 6522. [CrossRef]

${ }^{63}$ Gilbert, K. E.; Borden, W. T. Peracid oxidation of aliphatic amines: General synthesis of nitroalkanes. The Journal of Organic Chemistry 1979, 44, 659. [CrossRef]

${ }^{64}$ Tollari, S.; Porta, F. Stoichiometric and catalytic oxidation of cyclohexylamine by $\mathrm{Mo}(\mathrm{O})\left(\mathrm{O}_{2}\right)_{2}\left(\mathrm{H}_{2} \mathrm{O}\right)(\mathrm{HMPA})$. Journal of Molecular Catalysis 1993, 84, L137. [CrossRef]

${ }^{65}$ Kidwai, M.; Bhardwaj, S. Transformation of amines to oximes using heterogeneous nanocrystalline titanium (IV) oxide as a green catalyst. Synthetic Communication 2011, 41, 2655. [CrossRef]

${ }^{66}$ Suzuki, K.; Watanabe, T.; Murahashi, S.-I. Oxidation of primary amines to oximes with molecular oxygen using 1,1-diphenyl-2picrylhydrazyl and $\mathrm{WO}_{3} / \mathrm{Al}_{2} \mathrm{O}_{3}$ as catalysts. The Journal of Organic Chemistry 2013, 78, 2301. [CrossRef] [PubMed]

${ }^{67}$ Hwu, J. R.; Tseng, W. N.; Patel, H. V.; Wong, F. F.; Horng, D.-N.; Liaw, B. R.; Lin, L. C. Monodeoxygenation of nitroalkanes, nitrones, and heterocyclic $\mathrm{N}$-oxides by hexamethyldisilane through 1,2-elimination: Concept of "counterattack" reagent. The Journal of Organic Chemistry 1999, 64, 2211. [CrossRef]

${ }^{68}$ Chow, Y. L. Nitrosamine photochemistry: Reactions of aminium radicals. Accounts of Chemical Research 1973, 6, 354. [CrossRef]

${ }^{69}$ Akhtar, M.; Pechet, M. M. The mechanism of the Barton reaction. Journal of the American Chemical Society 1965, 86, 265. [CrossRef]
${ }^{70}$ Grossman, R. B. The Art of Writing Reasonable Organic Reaction Mechanisms, 2a. ed., Springer, 2002, p.253.

${ }^{71}$ Wittcoff, H. A.; Reuben, B. G.; Plotkin, J. S. Industrial Organic Chemicals, 2a. ed., John Wiley \& Sons - Canadá, 2004, cap. 7.

72 Murahashi, S.-I.; Mitsui, H.; Shiota, T.; Tsuda, T.; Watanabe, S. Tungstate-catalyzed oxidation of secondary amines to nitrones. $\alpha$ Substitution of secondary amines via nitrones. The Journal of Organic Chemistry 1990, 55, 1736. [CrossRef]

${ }^{73}$ Melo, J. O. F.; Donnici, C. L.; Augusti, R.; Ferreira, V. F.; Souza, M. C. B. V.; Ferreira, M. L. G.; Cunha, A. C. Heterociclos 1,2,3triazólicos: histórico, métodos de preparação, aplicações e atividades farmacológicas. Química Nova 2006, 29, 569. [CrossRef]

${ }^{74}$ Tavares, A.; Schneider, P. H.; Merlo, A. A. 3,5-disubstituted isozaxolines as potential molecular kits for liquid-crystalline materials. European Journal of Organic Chemistry 2009, 6, 889. [CrossRef]

${ }^{75}$ Tavares, A.; Schneider, P. H.; Merlo, A. A. 3-Arylisoxazolyl-5-carboxylic acid and 5(hydroxymethyl)-3-aryl-2-isoxazoline as molecular platforms for liquid-crystalline materials. Journal of the Brazilian Chemical Society 2009, 20, 1752. [CrossRef]

${ }^{76}$ Beckmann, E. Ueber die reaction zur umlagerung von oximido verbindungen in amide. Berichte der Deutschem Chemischem Gesellschaft 1894, 27, 300. [CrossRef]

${ }^{77}$ Hill, R.; Chortyk, O. T. Stereoisomerization of migrating group during Beckmann Rearrangement. Journal of the American Chemical Society 1962, 86, 1064. [CrossRef]

${ }^{78}$ Rai, K. M. L. Em Synthesis of heterocycles via cycloadditions II; Gupta, R. R., ed.; Springer, 2008, cap.1

${ }^{79}$ Gothelf, K. V. Em Cycloaddition reactions in organic synthesis; Kobayashi, S.; Jorgensen, K. A., eds.; Wiley-VCH Verlag, 2001, cap. 6.

${ }^{80}$ Gothelf, K. V.; Jorgensen, K. A. Asymmetric 1,3-dipolar cycloaddition reactions. Chemical Reviews 1998, 98, 863. [CrossRef] [PubMed] ${ }^{81}$ Eicher, T.; Hauptmann, S.; Speicher, A. The chemistry of heterocycles. Structure, reactions, syntheses, and applications, $2 \mathrm{a}$. ed., Wiley-VCH, 2003. 
${ }^{82}$ Belen'kii, L. I. Em Nitrile oxides, nitrones and nitronates in organic synthesis: Novel Strategies in Synthesis; Feuer, H., ed.; 2a ed., John Wiley \& Sons: New York, 2007, cap. 1.

${ }^{83}$ Freeman, J. P. Less familiar reactions of oximes. Chemical Reviews, 1973, 73, 283. [CrossRef]

${ }^{84}$ Diekmann, H.; Lüttke, W. A. A productive method for the preparation of geminal chloronitroso compounds. Angewandte Chemie International Edition 1968, 7, 387. [CrossRef]

${ }^{85}$ Wieser, K.; Berndt, A. Stable 4H-1,2oxazete by intramolecular cyclization of [?], [?unsaturated nitroso compound. Angewandte Chemie International Edition 1975, 14, 70. [CrossRef]

${ }^{86}$ Faragher, R.; Gilchrist, T. L. Cycloaddition reactions of nitrosoalkenes and azoalkenes with cyclopentadiene and other dienes. Journal of the Chemical Society, Perkin Transaction 1 1979, 249. [CrossRef]

${ }^{87}$ Dornow, A.; Jordan, H. D. Die verwendung von ?-chlor-oximen zur darstellung hoherer oxime. Chemische Berichte 1961, 94, 76. [CrossRef]

${ }^{88}$ Gilchrist, T. L. Nitroso-alkenes and nitrosoalkynes. Chemical Society Reviews 1983, 12, 53. [CrossRef]

${ }^{89}$ Olah, G. A.; Ramaiah, P.; Lee, C. S.; Prakash, G. K. S. Convenient oxidation of oximes to nitro compounds with sodium perborate in glacial acetic acid. Synlett 1992, 337. [CrossRef]

${ }^{90}$ Ballistreri, P.; Barbuzzi, E.; Tomaselli, G. A.; Toscano, R. M. Useful oxidation procedure of oximes to nitro compounds with Benz-Mo in acetonitrile. Synlett 1996, 1093. [CrossRef]

${ }^{91}$ Zon, J. Synthesis of diisopropyl 1nitroalkanephosphonates from diisopropyl 1oxoalkanephosphonates. Synthesis 1984, 661. [CrossRef]

92 Corey, E. J.; Estreicher, H. Nitrodestannylation. A new synthesis of conjugated nitro cyclo olefins. Tetrahedron Letters 1980, 21, 1113. [CrossRef]

${ }^{93}$ Foley, P. J. A novel method of converting aldehydes into nitriles under mild conditions. The reaction of dialkyl hydrogen phosphonates with oximes. The Journal of Organic Chemistry 1969, 34, 2805. [CrossRef] ${ }^{94}$ Dennis, W. E. Nitrile synthesis. The dehydration of amides by silazanes, chlorosilanes, alkoxysilanes, and aminosilanes. The Journal of Organic Chemistry 1970, 36, 3253. [CrossRef]

${ }^{95}$ Rosini, G.; Baccolini, G.; Cacchi, S. Nitriles from aldoximes. A new reaction of phosphonitrilic chloride. The Journal of Organic Chemistry 1973, 38, 1060. [CrossRef]

${ }^{96}$ Suzuki, H.; Fuchita, T.; Iwasa, A.; Mishina, T. Diphosphorus tetraiodide as a reagent for converting epoxides into olefins, and aldoximes into nitriles under mild conditions. Synthesis 1978, 905. [CrossRef]

${ }^{97}$ Rasmussen, J. K. Crown ethers in organic synthesis II. Catalytic dehydration of aldoximes. Chemistry Letters 1977, 6, 1295. [CrossRef]

${ }^{98}$ Keumi T., Yamamoto T., Saga H., Kitajima $\mathrm{H}$. Bull. N-trifluoroacetylimidazoles as a reagent for converting aldoximes into nitriles under mild conditions. The Chemical Society of Japan 1981, 54, 1579. [CrossRef]

${ }^{99}$ Kim, S.; Yi, K. Y. 1,1'-Thiocarbonyldi-2,2'pyridone. A new useful reagent for functional group conversions under essentially neutral conditions. The Journal of Organic Chemistry 1986, 51, 2615. [CrossRef]

${ }^{100}$ Kaneda, K.; Doken, K.; Imanaka, T. High deoxygenation ability of Rh carbonyl clusters toward oximes leading to nitrile formation. Chemistry Letters 1988, 285. [CrossRef]

${ }^{101}$ Yang, S. H.; Chang, S. Highly efficient and catalytic conversion of aldoximes to nitriles. Organic Letters 2001, 3, 4209. [CrossRef] [PubMed]

${ }^{102}$ Sharghi, H.; Sarvari, M. H. A direct synthesis of nitriles and amides from aldehydes using dry or wet alumina in solvent free conditions. Tetrahedron 2002, 58, 10323. [CrossRef]

${ }^{103}$ Boruah, $\quad$ M.; Konwa, D. $\mathrm{AlCl}_{3} \bullet 6 \mathrm{H}_{2} \mathrm{O} / \mathrm{KI} / \mathrm{H}_{2} \mathrm{O} / \mathrm{CH}_{3} \mathrm{CN}$ : A new alternate system for dehydration of oximes and amides in hydrated media. The Journal of Organic Chemistry 2002, 67, 7138. [CrossRef] [PubMed] 
${ }^{104}$ Olah, G. A.; Narang, S. C.; Garcia-Luna, A. Synthetic methods and reactions; $83^{1}$. Sulfuryl chloride fluoride, a mild dehydrating agent in the preparation of nitriles from aldoximes. Synthesis 1980, 659. [CrossRef]

${ }^{105}$ Attanasi, O.; Palma, P.; Serra-Zanetti, F. Effect of metal ions in organic synthesis; XVII. Mild, easy, and high-yield conversion of aldoximes into nitriles under copper(II) acetate-catalysis. Synthesis 1983, 741. [CrossRef]

${ }^{106}$ Konwar, D.; Boruah, R. C.; Sandhu, J. S. A facile dehydration and Beckmann rearrangement of oximes with aluminium iodide. Tetrahedron Letters 1990, 31, 1063. [CrossRef]

$107 \mathrm{Li}, \quad$ Z.; Lu, Z. Silica-supported dichlorophosphate catalyzed Beckmann rearrangement and dehydration of oximes under microwave irradiation. Letters in Organic Chemistry 2008, 5, 495. [CrossRef]

${ }^{108}$ Yadav, L. D. S.; Srivastava, V. P.; Patel, R. Bromodimethylsulfonium bromide (BDMS): a useful reagent for conversion of aldoximes and primary amides to nitriles. Tetrahedron Letters 2009, 50, 5532. [CrossRef]

${ }^{109}$ Moussa, Z.; Ahmed, S. A.; EIDouhaibi, A. S.; Al-Raqa, S. Y. NMR Studies and electrophilic properties of triphenylphosphine-trifluoromethanesulfonic anhydride; a remarkable dehydrating reagent system for the conversion of aldoximes into nitriles. Tetrahedron Letters 2010, 51, 1826. [CrossRef]

${ }^{110}$ Wang, S. S.; Sukenik, C. N. The reduction of oximes by lithium aluminum hydride in hexaphosphoramide solvent. The Journal of Organic Chemistry 1985, 50, 5448. [CrossRef]

${ }^{111}$ Abiraj, K.; Gowda, D. C. Magnesiumcatalyzed proficient reduction of oximes to amines using ammonium formate. Synthetic Communications 2004, 34, 599. [CrossRef] 112 Inffland, D. C.; Yen, T.-F. Preparation of amines by low pressure hydrogenation of oximes. Journal of the American Chemical Society 1954, 76, 4180. [CrossRef]

${ }^{113}$ Huang, X.; Ortiz-Marciales, M.; Huang, K.; Stepanenko, V.; Merced, F. G.; Ayala, A. M.; Correa, W.; De Jesús, M. Asymmetric synthesis of primary amines via the spiroborate-catalyzed borane reduction of oxime ethers. Organic Letters 2007, 9, 1793. [CrossRef] [PubMed]

${ }^{114}$ Itsuno, S.; Tanaka. K.; Ito, K. Asymmetric reduction of chiral acetophenone oxime ethers to optically active primary amines. Chemistry Letters 1986, 15, 1133. [CrossRef]

${ }^{115}$ Takei, I.; Nishibayashi, Y.; Ishii, Y.; Mizobe, Y.; Uemura, S.; Hidai, M. Rutheniumcatalysed asymmetric hydrosilylation of ketoximes using chiral oxazolinyl ferrocenylphosphines. Chemical Communication 2001, 22, 2360. [CrossRef] ${ }^{116}$ Sasse, A.; Ligneau, X.; Rouleau, A.; Elz, S.; Ganellin, C. R.; Arrang, J.-M.; Schwartz, J.-C.; Schunack, W.; Stark, H. Influence of bulky substituents on histamine $\mathrm{H} 3$ receptor agonist/antagonist properties. Journal Medicinal Chemistry 2002, 45, 4000. [CrossRef] [PubMed] 